LAWRENCE LIVERM ORE N A TIO NAL LABORATORY

\section{UV Excited Photoacoustic Raman}

J. Carter, D. Chambers, P. Steele, P. Haugen, D. Heller

November 26, 2013 
This document was prepared as an account of work sponsored by an agency of the United States government. Neither the United States government nor Lawrence Livermore National Security, LLC, nor any of their employees makes any warranty, expressed or implied, or assumes any legal liability or responsibility for the accuracy, completeness, or usefulness of any information, apparatus, product, or process disclosed, or represents that its use would not infringe privately owned rights. Reference herein to any specific commercial product, process, or service by trade name, trademark, manufacturer, or otherwise does not necessarily constitute or imply its endorsement, recommendation, or favoring by the United States government or Lawrence Livermore National Security, LLC. The views and opinions of authors expressed herein do not necessarily state or reflect those of the United States government or Lawrence Livermore National Security, LLC, and shall not be used for advertising or product endorsement purposes.

This work performed under the auspices of the U.S. Department of Energy by Lawrence Livermore National Laboratory under Contract DE-AC52-07NA27344. 


\title{
UV Excited Photoacoustic Raman Spectroscopy
}

\author{
J. Chance Carter, David H. Chambers, Paul T. Steele, Peter Haugen, Don Heller
}

\section{Introduction}

Photoacoustic Raman spectroscopy[1] (PARS) is a method of detecting low concentrations of target analytes in gas mixtures from the inelastic scattering of laser light. Two lasers with slightly different wavelengths are focused at the same point. If the wavelength difference corresponds to the transition energy between two molecular vibrational levels of a target analyte, energy is transferred from one wavelength (pump) to the other (Stokes). In the process, the molecules are left in an excited state that subsequently decays back to the ground state through collisions with the gas, producing heat. The sudden heating creates acoustic waves that can be detected by a microphone or other acoustic detector. In this article we derive a model of the Raman scattering process coupled with the generation of acoustic waves. The goal of the model is to predict the acoustic pulse shape and intensity from knowledge of the laser pulses and target analyte concentration. Model calculations can then be used to optimize receiver designs and laser characteristics and improve overall system performance.

The rate of Stokes beam amplification depends on the intensities of the pump laser beam and the initial Stokes beam. The theory of the Raman scattering process for PARS is well described in the review paper by Barrett[1]. The Raman scattering process leaves the optically active molecules in the gas (or liquid) in an energetic state that relaxes to the original state through collisions with the gas molecules. The resulting heating causes the gas to expand, exciting an acoustic wave that propagates away from the heated volume. The theory of acoustic wave generation from heating can be found in standard acoustic texts (see Morse and Ingard[2]). Application to photoacoustic absorption spectroscopy (PAS) can be found in the text by Lyamshev[3] and the article by Yonak and Dowling[4] and references therein. In most previous applications of PARS the lasers were focused into a closed gas cell containing the target analyte. A microphone emplaced in the cell wall was used to detect the acoustic signal. The signal was amplified by modulating the laser intensities at a rate that matched an acoustic resonant frequency of the cell. Barrett in his early work used a quasi-steady acoustic model to calculate the acoustic response of a cell. Das et al[5] constructed a dynamic model of the acoustic signal to estimate the signal and noise powers for particulate analytes. In this article we combine a dynamic Raman scattering model found in Barrett[1] with the time-dependent acoustic pulse generation model from Yonak and Dowling[4].

\section{Methods}

\subsection{Dynamic Raman scattering and heat deposition model}

In this section we combine the dynamic two-level Raman scattering model from Barrett[1] with Gaussian intensity distributions for the pump and Stokes beams to obtain an expression for the rate of heat deposition during the Raman process. Raman scattering is a process where two laser beams with different wavenumbers interact nonlinearly to draw energy from the pump beam to amplify the Stokes beam. The process occurs when the 
difference in wavenumbers matches the difference in energy levels between two states of a molecule in the propagating medium. The condition for Raman conversion is $\Delta E=h c\left(1 / \lambda_{p}-1 / \lambda_{s}\right)$, where $\Delta E$ is the difference in energy between the two states, $\lambda_{p}$ is the pump wavelength, $\lambda_{s}$ is the Stokes wavelength, $c$ is the speed of light, and $h$ is Planck's constant. The interaction occurs in the region where the pump and Stokes beams overlap. Within this region the intensity of the Stokes beam grows by a factor of $e^{g_{s} z}-1$, where $z$ is the distance down the Stokes beam axis in the interaction region and $g_{s}$ is the Raman gain. If the gain is weak and the interaction region short we can approximate this growth factor with $g_{s} z$.

In the two level dynamic Raman model we assume only two energy states (levels) are involved in the interaction process. Raman scattering induces molecules to transition from the lower to upper energy state. Intermolecular collisions and spontaneous emission cause the excited molecules to lose energy and move back to the lower state. If $N_{0}(t)$ is the number of molecules per unit volume in the lower ground state and $N_{1}(t)$ is the number per unit volume in the upper excited state at time $t$, the rate of change of molecules in the excited state is

$$
\frac{d N_{1}}{d t}=k_{r} N_{0}+k_{c}^{0} N_{0}-k_{r} N_{1}-k_{c}^{1} N_{1}-k_{e} N_{1}
$$

where $k_{r}$ is the rate of Raman transitions, $k_{c}^{0}$ is the rate of collision-induced transitions from lower to upper states, $k_{c}^{1}$ is the rate of collision-induced transitions from upper to lower states, and $k_{e}$ is the rate of spontaneous emission from upper to lower states. In the absence of Raman scattering we assume the populations of the states are in thermal equilibrium with $N_{1}=N_{1}^{e}=N_{0}^{e} e^{-\Delta E / k_{b} T}$, with $k_{b}$ Boltzman's constant and $T$ the absolute temperature. The equilibrium populations are constant $\left(d N_{1}^{e} / d t=0\right)$ which implies a balance between collision-induced transitions and spontaneous emission: $k_{c}^{0} N_{0}^{e}=\left(k_{c}^{1}+k_{e}\right) N_{1}^{e}$. The total number of molecules per unit volume $N$ is conserved, i.e. $N=N_{0}+N_{1}=N_{0}^{e}+N_{1}^{e}$. Following Barrett, we can combine these relationships into an equation for the evolution of the excess population due to Raman scattering $F_{1}=N_{1}-N_{1}^{e}$ :

$$
\frac{d F_{1}}{d t}=C_{1}-C_{2} F_{1}
$$

The coefficients $C_{1}$ and $C_{2}$ are given by

$$
\begin{aligned}
& C_{1}=k_{r}\left(1-e^{\Delta E / k_{b} T}\right) N_{0}^{e}, \\
& C_{2}=2 k_{r}+k_{c}^{1}+\left(k_{c}^{1}+k_{e}\right) e^{-\Delta E / k_{b} T} .
\end{aligned}
$$

The collision transition rate $k_{c}^{1}$ and spontaneous emission rate $k_{e}$ can be regarded as constant to a good approximation. However, the Raman transition rate $k_{r}$ depends on the intensities of the pump $\left(I_{p}\right)$ and Stokes $\left(I_{s}\right)$ beams, $k_{r}=C_{0} I_{s}(\mathbf{x}, t) I_{p}(\mathbf{x}, t)$ (weak gain limit), which can vary with position $\mathbf{x}$ and time $t$. The proportionality constant $C_{0}$ is 


$$
C_{0}=\frac{2 \lambda_{s}^{4}}{h^{2} c^{2} n_{s} n_{p} \Gamma}\left(\frac{d \sigma}{d \Omega}\right),
$$

where $n_{s}$ and $n_{p}$ are the refractive indices of the medium at the Stokes and pump wavelengths, $\Gamma$ is the full-width half-maximum of the spontaneous Raman spectral line, and $(d \sigma / d \Omega)$ is the differential cross-section for spontaneous Raman scattering. If the pump and Stokes lasers are $\mathrm{cw}$ and turned on at $t=0$, we can solve the evolution equation (2) for $t>0$ :

$$
F_{1}(\mathbf{x}, t)=\frac{C_{1}(\mathbf{x})}{C_{2}(\mathbf{x})}\left(1-e^{-C_{2}(\mathbf{x}) t}\right) .
$$

Both $C_{1}(\mathbf{x})$ and $C_{2}(\mathbf{x})$ may be complicated functions of position $\mathbf{x}$ depending on the beam geometry and intensity profiles. If the $\mathrm{cw}$ beams are turned off at time $t=t_{1}>0$, then $F_{1}(t)$ for $t>t_{1}$ will decay like

$$
F_{1}(\mathbf{x}, t)=\frac{C_{1}(\mathbf{x})}{C_{2}(\mathbf{x})}\left(1-e^{-C_{2}(\mathbf{x}) t_{1}}\right) e^{-C_{3}\left(t-t_{1}\right)}
$$

where

$$
C_{3}=k_{c}^{1}+\left(k_{c}^{1}+k_{e}\right) e^{-\Delta E / k_{b} T} .
$$

In most applications, the thermal energy of the molecules is much smaller than the transition energy $\left(k_{b} T=\Delta E\right)$ and the rate of spontaneous emission can be neglected $\left(k_{e}=k_{c}^{1}\right)$. Then the $C_{n}$ terms can be approximated as

$$
C_{1}(\mathbf{x}, t) \approx k_{r}(\mathbf{x}, t) N, C_{2}(\mathbf{x}, t) \approx 2 k_{r}(\mathbf{x}, t)+k_{c}^{1}, C_{3} \approx k_{c}^{1} .
$$

All molecules in the upper energy state eventually transition back down to the lower state. The energy of those that decay by collisions with neighboring molecule appears as heat. The amount of heat produced per unit volume is $H(\mathbf{x}, t)=\Delta E k_{c}^{1} F_{1}(\mathbf{x}, t)$. The spatial distribution of the heating depends on the spatial distributions of the pump beam and Stokes beam intensities. A particularly simple beam configuration is for both pump and Stokes beams to be Gaussian with a common axis and focus size. If we select the $z$ axis to be along the beam with the origin at the focus, the intensity distribution for the pump beam is

$$
\begin{aligned}
& I_{p}^{G}(\mathbf{x})=I_{p 0} \frac{D_{m}^{2}}{D_{p}^{2}(z)} e^{-8 r^{2} / D_{p}^{2}(z)}, \\
& \frac{D_{p}^{2}(z)}{D_{m}^{2}}=1+\frac{16 \lambda_{p}^{2} z^{2}}{\pi^{2} D_{m}^{4}}
\end{aligned}
$$

where $D_{m}$ is the focus width. The intensity distribution for the Stokes beam is written similarly.

Though the intensity distribution for a Gaussian beam is relatively simple, it still gives a complicated heating pattern since it appears in the argument of the exponential in our expressions for $F_{1}$. The best type of intensity distribution for modeling would be one that is constant along a finite length of beam axis and zero everywhere else. We can create such a distribution in a way that approximates a Gaussian beam with low divergence. 
Consider the integral of the product of pump and Stokes beam intensities in a plane perpendicular to the beam axis:

$$
\int_{0}^{\infty} I_{p}^{G}(r, z) I_{s}^{G}(r, z) r d r=\frac{\pi D_{m}^{2}}{16} \frac{I_{p 0} I_{s 0}}{1+8 z^{2}\left(\lambda_{p}^{2}+\lambda_{s}^{2}\right) / \pi^{2} D_{m}^{4}} .
$$

This has a maximum of $\pi D_{m}^{2} I_{p 0} I_{s 0} / 4$ at the focus $z=0$, and falls off like $1 / z^{2}$ at large distances from it. We will replace the product of Gaussian intensity distribution with the following:

$$
I_{p}(\mathbf{x}) I_{s}(\mathbf{x})=\frac{\pi}{16} D_{m}^{2} I_{p 0} I_{s 0} U\left(\frac{L_{e}}{2}-|z|\right) \delta(x) \delta(y),
$$

where $U(\cdot)$ is the unit step function. The effective length $L_{e}$ is determined by requiring

$$
\int_{-\infty}^{\infty} \int_{0}^{\infty} I_{p}^{G}(r, z) I_{s}^{G}(r, z) r d r d z=\frac{\pi}{16} D_{m}^{2} L_{e} I_{p 0} I_{s 0} .
$$

The resulting expression for $L_{e}$ is

$$
L_{e}=\frac{\pi^{2} D_{m}^{2}}{\sqrt{2\left(\lambda_{p}^{2}+\lambda_{s}^{2}\right)}}
$$

This completes the specification of the piecewise constant model for the intensity product that will be used to calculate the acoustic response to the heating from PARS. The final expression for the heating distribution is

$$
H(\mathbf{x}, t)=\Delta E k_{c}^{1} F_{1}(t) \delta(x) \delta(y) U\left(\frac{L_{e}}{2}-|z|\right) .
$$

The function $F_{1}(t)$ is the same as before.

\subsection{Acoustic pulse generation}

Given the heat distribution $H(\mathbf{x}, t)$ the acoustic pressure pulse $p(\mathbf{x}, t)$ is obtained from solving $[2,3,4]$

$$
\frac{1}{c_{s}^{2}} \frac{\partial^{2} p}{\partial t^{2}}-\nabla^{2} p=\frac{\alpha}{C_{p}} \frac{\partial H}{\partial t}
$$

where $c_{s}$ is the sound speed, $\alpha$ is the coefficient of thermal expansion, and $C_{p}$ is the heat capacity at constant pressure. Using the Green's function $G(\mathbf{R}, t)=\delta\left(t-R / c_{s}\right) / 4 \pi R$ (where $R=|\mathbf{R}|)$ the solution of this equation can be written

$$
p(\mathbf{x}, t)=\frac{\alpha}{4 \pi C_{p}} \int \frac{\partial}{\partial t} H\left(\mathbf{x}^{\prime}, t-\frac{\left|\mathbf{x}-\mathbf{x}^{\prime}\right|}{c_{s}}\right) \frac{d \mathbf{x}^{\prime}}{\left|\mathbf{x}-\mathbf{x}^{\prime}\right|} .
$$

The volume integral is over the region of support of $H(\mathbf{x}, t)$. For the two-level Raman model, the time derivative of the heat distribution (equation 16) has the general form

$$
\frac{\partial H}{\partial t}=A e^{-\beta(t-\tau)} \delta(x) \delta(y) U\left(\frac{L_{e}}{2}-|z|\right) .
$$

The quantities $A, \beta$, and $\tau$ have different values depending on the time interval. For $t<0$, $A=0$ and the other quantities are left undefined. Otherwise $A=A_{1}, \beta=\left(k_{c}^{1}+2 k_{r}\right), \tau=0$ 
for $0 \leq t<t_{1}$, or $A=A_{2}, \quad \beta=k_{c}^{1}, \tau=t_{1}$ for $t \geq t_{1}$. The $A_{n}$ constants are

$$
A_{1}=\Delta E k_{r} k_{c}^{1} N, \quad A_{2}=-\Delta E\left(k_{c}^{1}\right)^{2} \frac{k_{r} N}{k_{c}^{1}+2 k_{r}}\left[1-e^{-\left(k_{c}^{1}+2 k_{r}\right) t_{1}}\right] .
$$

Substituting the general form of equation (19) into the integral for the pressure field, equation (18), we obtain

$$
p(r, z, t)=\frac{\alpha A}{4 \pi C_{p}} \int_{-L_{e} / 2}^{L^{\prime} / 2} \frac{d z^{\prime}}{R\left(z^{\prime}\right)} U\left(t-\tau-R\left(z^{\prime}\right) / c_{s}\right) e^{-\beta\left(t-\tau-R\left(z^{\prime}\right) / c_{s}\right)},
$$

where $R\left(z^{\prime}\right)=\sqrt{r^{2}+\left(z-z^{\prime}\right)^{2}}, \quad r=\sqrt{x^{2}+y^{2}}$. The integrand is composed of three factors: the exponential decay that describes the Raman dynamics, the step function which enforces causality (zero contribution until the time $t-\tau$ is greater than the travel time $R / c_{s}$ ), and the geometric spreading factor $1 / R$. The domain of integration is the length of the laser interaction region. Each length segment contributes an exponential decay factor weighted by the inverse distance between the segment and the observation point. The sum of the weighted and delayed contributions of each line segment generates the response at the observation point.

The pressure field given by equation (21) is symmetric around $z=0$ so we only need to evaluate the integral for $z \geq 0$. The most obvious approach is to replace the integration over $z^{\prime}$ with integration over $R$ with the substitution $d z^{\prime} / R\left(z^{\prime}\right)=d R / \sqrt{R^{2}-r^{2}}$. This greatly simplifies the integrand but complicates the range of integration. If the observation point is above the line segment $\left(z>L_{e} / 2\right)$, the function $R\left(z^{\prime}\right)$ is monotonic and the range of integration is from the closest endpoint $R_{\min }=R\left(L_{e} / 2\right)$ to the farthest endpoint $R_{\max }=R\left(-L_{e} / 2\right)$ of the line segment (Fig. 1 left). If the observation point is in the region normal to the line segment, $0 \leq z \leq L_{e} / 2$, the closest source point is $z^{\prime}=z$ in the middle of the source region with $R(z)=r$ (Fig. 1 right). The pressure field integral divides into a contribution from the line segment from $z^{\prime}=2 z-L_{e} / 2$ to $z^{\prime}=L_{e} / 2\left(R=r\right.$ to $\left.R=R_{\min }\right)$, and a contribution from the remaining segment $\left(R=R_{\min }\right.$ to $R=R_{\max }$ ). In the first segment, there are two disjoint source points with the same geometric distance from the observation point, effectively doubling the strength of the wave generated from that segment. The effect of the step function in the integrand is to further restrict the range of integration to values of $R$ less than $c_{s}(t-\tau)$, i.e. no source point on the line segment can contribute to the field at the observation point until the elapsed time exceeds the travel time between the two points. The combination of geometry and causality produces different expressions for the field depending on combinations of elapsed time $t-\tau$ and vertical location $z$. However, in each case the field is obtained by evaluating linear combinations of terms with the general form

$$
\begin{aligned}
p_{g}(r, z, t) & =\frac{\alpha A}{4 \pi C_{p}} e^{-\beta(t-\tau)} \int_{r}^{R_{0}} \frac{d R}{\sqrt{R^{2}-r^{2}}} e^{\beta R / c_{s}}, \\
& =\frac{\alpha A}{4 \pi C_{p}} e^{-\beta(t-\tau)} \int_{1}^{R_{0} / r} \frac{d s}{\sqrt{s^{2}-1}} e^{\beta r s / c_{s}},
\end{aligned}
$$




$$
=\frac{\alpha A}{4 \pi C_{p}} e^{-\beta(t-\tau)} f\left(\frac{\beta r}{c_{s}}, \frac{R_{0}}{r}\right),
$$

where $R_{0} / r \geq 1$. The function $f$ is

$$
f(x, y)=\int_{1}^{y} \frac{d s}{\sqrt{s^{2}-1}} e^{x s} .
$$

For the field in the region $|z|>L_{e} / 2$, we have

$$
p(r, z, t)=\frac{\alpha A}{4 \pi C_{p}} e^{-\beta(t-\tau)}\left\{\begin{array}{cc}
0, & t-\tau<\frac{R_{\min }}{c_{s}} \\
f\left(\frac{\beta r}{c_{s}}, \frac{c_{s}(t-\tau)}{r}\right)-f\left(\frac{\beta r}{c_{s}}, \frac{R_{\min }}{r}\right), & \frac{R_{\min }}{c_{s}} \leq t-\tau<\frac{R_{\max }}{c_{s}} \\
f\left(\frac{\beta r}{c_{s}}, \frac{R_{\max }}{r}\right)-f\left(\frac{\beta r}{c_{s}}, \frac{R_{\min }}{r}\right), & t-\tau \geq \frac{R_{\max }}{c_{s}} .
\end{array}\right.
$$

Similarly for the field in the region $|z| \leq L_{e} / 2$ we obtain

$$
p(r, z, t)=\frac{\alpha A}{4 \pi C_{p}} e^{-\beta(t-\tau)}\left\{\begin{array}{cc}
0, & t-\tau<\frac{r}{c_{s}} \\
2 f\left(\frac{\beta r}{c_{s}}, \frac{c_{s}(t-\tau)}{r}\right), & \frac{r}{c_{s}} \leq t-\tau<\frac{R_{\min }}{c_{s}} \\
f\left(\frac{\beta r}{c_{s}}, \frac{R_{\min }}{r}\right)+f\left(\frac{\beta r}{c_{s}}, \frac{c_{s}(t-\tau)}{r}\right), & \frac{R_{\min }}{c_{s}} \leq t-\tau<\frac{R_{\max }}{c_{s}} \\
f\left(\frac{\beta r}{c_{s}}, \frac{R_{\min }}{r}\right)+f\left(\frac{\beta r}{c_{s}}, \frac{R_{\max }}{r}\right), & t-\tau \geq \frac{R_{\max }}{c_{s}} .
\end{array}\right.
$$

For most applications we are interested in the field far from the source region. This is also the limit implied by the simplifications that lead to the line segment model for the Raman active region. More specifically, if we restrict our attention to radial distances $r$ where the travel time of the pulse $r / c_{s}$ is much longer than the time scale for the Raman conversion, then $\beta r / c_{s}$ is large and we can use the asymptotic representation of $f(x, y)$ for large $x$ found in Appendix A, e.g.

$$
f\left(\frac{\beta r}{c_{s}}, \frac{R}{r}\right) ; \frac{c_{s}}{\sqrt{\beta}} e^{\beta R / c_{s}} \sqrt{\frac{c_{s}\left(R^{2}-r^{2}\right)}{c_{s}^{2} r^{2}+\beta^{2}\left(R^{2}-r^{2}\right)^{2}}} .
$$

Using this expression in equations (26) and (27), we can calculate the pulse emitted from the source region of size $L_{e}=7 \mathrm{~mm}$ and $D_{m}=40 \mu \mathrm{m}$ (see Appendix B). Figure 2 shows the emitted pressure pulse sampled along a line parallel to the source region at a radial distance of $r=L_{e}$. In the region from $z=0$ to $z=L_{e} / 2$ the primary feature is the cylindrical pulse moving normal to the source region. For $z>L_{e} / 2$ the pulse is spherical, radiating from the endpoint of the source region. This endpoint also generates a small pulse emitted into the 
$z<L_{e} / 2$ region that follows the main pulse. Figure 4 shows a detail of the structure of the main pulse. The shape is governed by the exponential behavior of the population of molecules in the upper Raman energy state. The initial rise of the pulse is determined by the growing population of molecules in the upper energy state during the laser pulse. At high gains, this is nearly instantaneous. After the end of the laser pulse, the decay of the pressure is set by the collisional relaxation rate and the length of the source region.
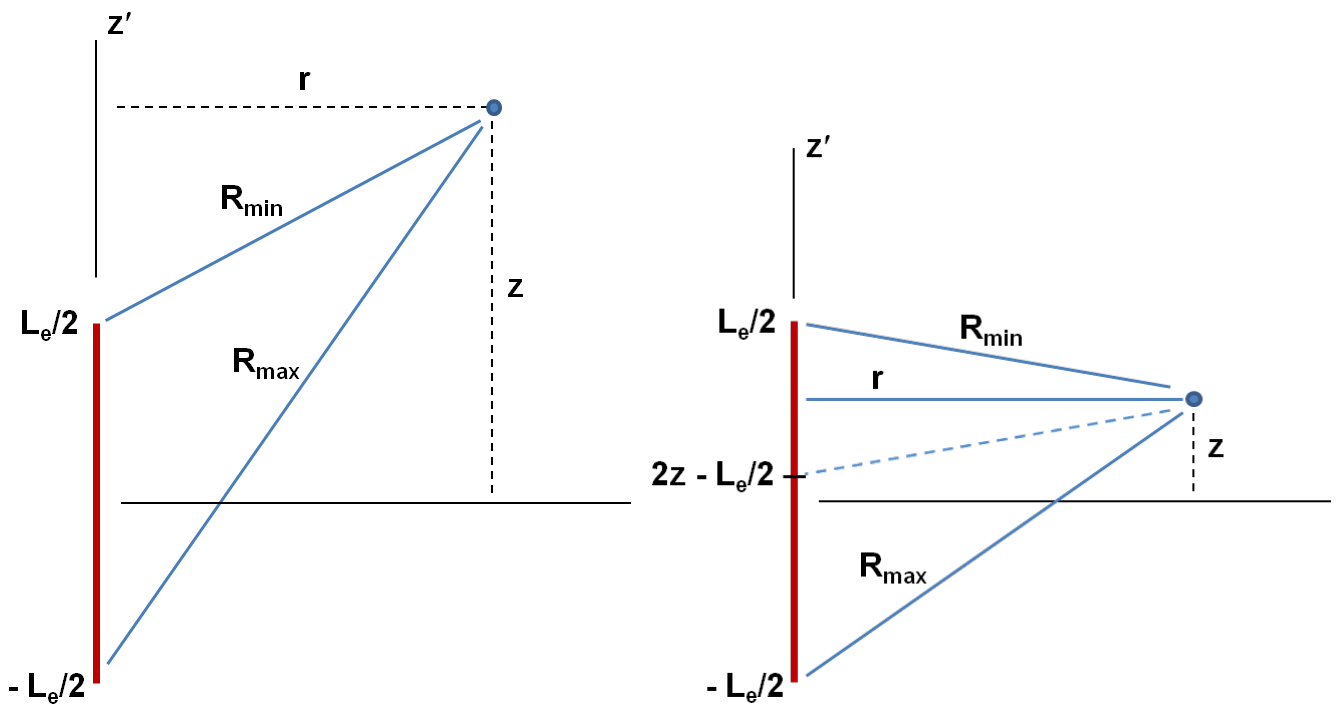

Figure 1: Geometry for evaluation of pressure field integral when observation point is above the source region (left) or normal to the source region (right).

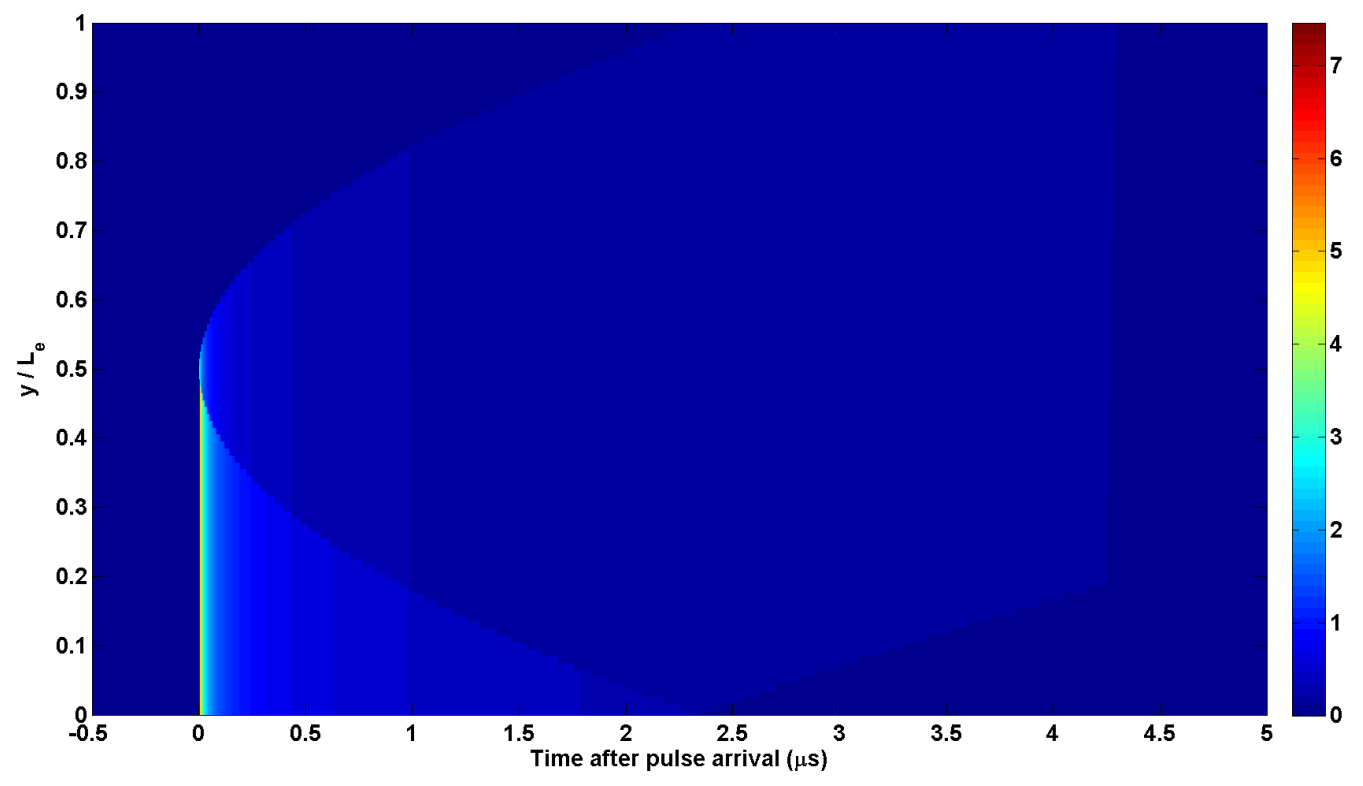

Figure 2: Pulse emitted from source region of length $L_{e}=7 \mathrm{~mm}$ and diameter $D_{m}=40 \mu \mathrm{m}$. Image shows time history of a pulse sampled along a line parallel to the source 
at a radial distance of one $L_{e}$. The time origin corresponds the the arrival time $L_{e} / c_{s}$ of the main pulse.

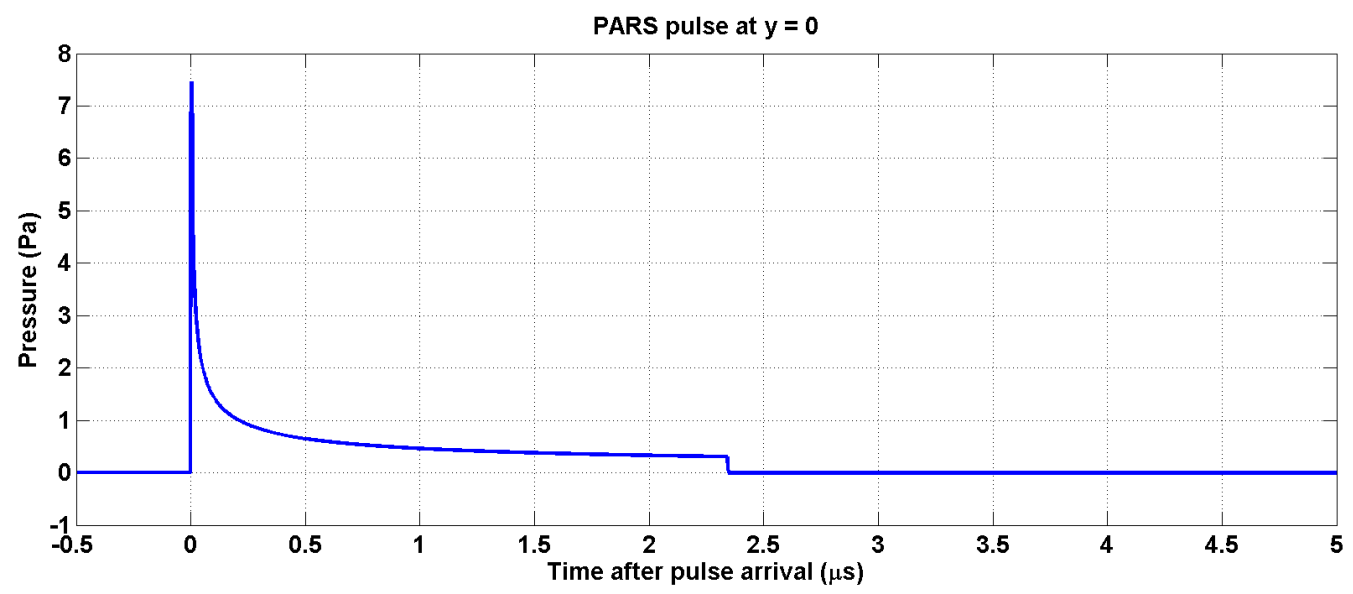

Figure 3: Detail of main pulse in Fig. 2 at $y=0$ showing the abrupt leading and trailing edge followed by an extended tail.

\subsection{Pulse generation with acoustic absorption}

Though formally correct, the above theory ignores acoustic absorption (or dissipation), which can be large at typical optical pulse times of microseconds or smaller. At these time scales, the acoustic absorption depends on the frequency, with the classical absorption coefficient $\alpha$ from viscosity and thermal conductivity increasing linearly with frequency[6]:

$$
\alpha_{c}=\frac{\pi \omega}{c_{s}^{2}}\left[\frac{4}{3} v+\beta_{H}\left(\gamma_{H}-1\right)\right],
$$

where $v$ is the kinematic viscosity, $\beta_{H}$ is the thermal diffusivity, and $\gamma_{H}$ is the ratio of specific heats. For our application, we use a binary mixture of hydrogen in nitrogen, which adds a mutual diffusivity contribution to the absorption:

$$
\alpha_{d}=\frac{\pi \omega}{c_{s}^{2}} \frac{\gamma_{H} D_{12}\left(M_{N}-M_{H}\right)^{2} V_{1}\left(1-V_{1}\right)}{\left[M_{N}\left(1-V_{H} M_{H} V_{H}\right]^{2}\right.},
$$

where $D_{12}$ is the mutual diffusivity, $M_{H}, M_{N}$ are the gram molecular weights of $H_{2}$ and $N_{2}$ respectively, and $V_{1}$ is the volume fraction of $H_{2}[6]$.

The linear frequency dependence of total absorption can be incorporated conveniently into the pulse generation problem. We begin by rewriting equation (21) for the pressure field as

$$
p(r, z, t)=\frac{\alpha A}{4 \pi C_{p}} \int_{-L_{e} / 2}^{L_{e} / 2} g_{0}\left(r, z, t ; z^{\prime}\right) d z^{\prime},
$$

where $g_{0}\left(r, z, t ; z^{\prime}\right)$ represents the integrand in equation (21). The temporal Fourier transform of $g$ is given by

$$
\hat{g}_{0}\left(r, z, \omega ; z^{\prime}\right)=\frac{1}{2 \pi} \int_{-\infty}^{\infty} g_{0}\left(r, z, t ; z^{\prime}\right) e^{i \omega t}
$$




$$
=-\frac{e^{i\left(k_{w} R\left(z^{\prime}\right)+\omega \tau\right)}}{2 \pi R\left(z^{\prime}\right)(i \omega-\beta)},
$$

with $k_{w}=\omega / c_{s}$ the wavenumber. Dissipation can be incorporated by adding an imaginary component to the wavenumber: $k_{w}=\omega / c_{s}+i \varepsilon \omega^{2} / c_{s} \quad\left(\varepsilon=c_{s}^{2} \alpha / 2 \pi \omega\right)$. A typical value for the dissipation constant $\varepsilon$ is $0.00018 \mathrm{~m} / \mathrm{s}^{2}$. This adds a factor of $\hat{h}(\omega)=\exp \left(-\varepsilon R\left(z^{\prime}\right) \omega^{2} / c_{s}^{3}\right)$ to $\hat{g}_{0}$. The inverse Fourier transform is

$$
\begin{aligned}
& g\left(r, z, t ; z^{\prime}\right)=\int_{-\infty}^{\infty} \hat{g}_{0}\left(r, z, \omega ; z^{\prime}\right) \hat{h}(\omega) e^{-i \omega t} d \omega \\
& =\frac{1}{2} \sqrt{\frac{c_{s}^{3}}{\pi \varepsilon R\left(z^{\prime}\right)}} \int_{-\infty}^{\infty} g_{0}\left(r, a, t^{\prime} ; z^{\prime}\right) e^{-c_{s}^{3}\left(t-t^{\prime}\right)^{2} / 4 \varepsilon R\left(z^{\prime}\right)} d t^{\prime} \\
& =\frac{1}{2 R\left(z^{\prime}\right)} \operatorname{erfc}\left(-\frac{1}{2} \sqrt{\frac{c_{s}^{3}}{\varepsilon R\left(z^{\prime}\right)}}\left[t-\tau-\left(1+\frac{2 \beta \varepsilon}{c_{s}^{2}}\right) \frac{R\left(z^{\prime}\right)}{c_{s}}\right]\right) e^{-\beta\left[t-\tau-\left(1+\frac{2 \beta \varepsilon}{c_{s}^{2}}\right) \frac{R\left(z^{\prime}\right)}{c_{s}}\right]} .
\end{aligned}
$$

The final expression for the pressure field with acoustic dissipation is

$$
p(r, z, t)=\frac{\alpha A}{8 \pi C_{p}} \int_{-L_{e} / 2}^{L_{e} / 2} \frac{d z^{\prime}}{R\left(z^{\prime}\right)} \operatorname{erfc}\left(-\frac{1}{2} \sqrt{\frac{c_{s}^{3}}{\varepsilon R\left(z^{\prime}\right)}}\left[t-\tau-\left(1+\frac{2 \beta \varepsilon}{c_{s}^{2}}\right) \frac{R\left(z^{\prime}\right)}{c_{s}}\right]\right) e^{-\beta\left[t-\tau-\left(1+\frac{2 \beta \varepsilon}{c_{s}^{2}}\right) \frac{R\left(z^{\prime}\right)}{c_{s}}\right]} .
$$

In the previous case (no dissipation), the step function in the integrand (eqn. (21)) lead to a set of expressions for the pressure field (eqns. (26) and (27)) that changed forms at different time intervals. In the dissipation case, the step function in the integrand has been replaced by the complementary error function. This reduces to a step function in the limit $\varepsilon \rightarrow 0$. For finite $\varepsilon$, the complementary error function smoothes the transitions between different intervals and broadening the overall pulse shape.

Figure 4 shows the pulse emitted from a source region of size $L_{e}=7 \mathrm{~mm}$ and $D_{m}=40 \mu \mathrm{m}$. The conditions are identical to those of the previous case except the Raman gain is only $1 \%$ the original value to avoid a numerical instability that occurs for high gains. The dissipation significantly smooths the emitted pulse as seen in Figure 5. 


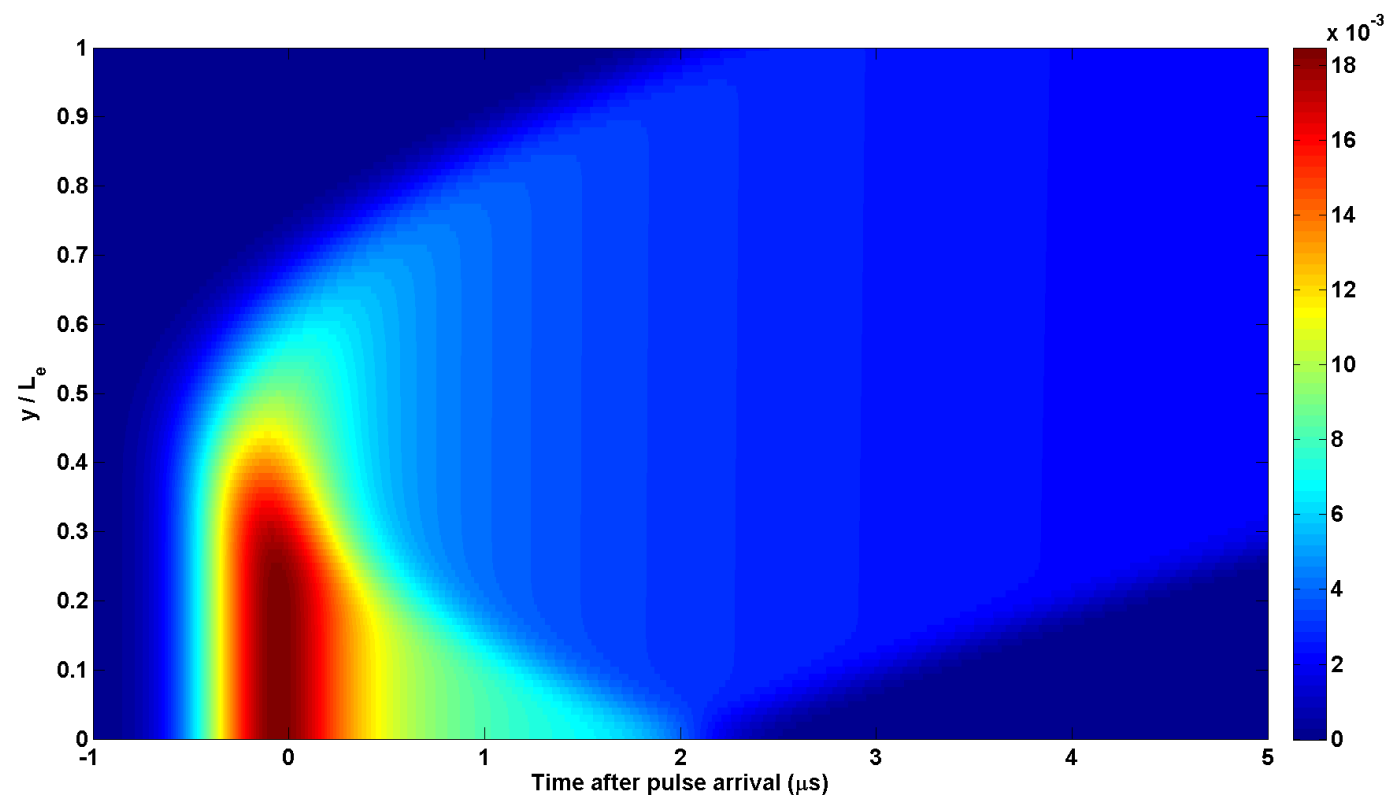

Figure 4: Pulse emitted from source region of length $L_{e}=7 \mathrm{~cm}$ and diameter $D_{m}=40 \mu \mathrm{m}$ with dissipation constant $\varepsilon=0.0018 \mathrm{~m}^{2} / \mathrm{s}$. Image shows time history of a pulse sampled along a line parallel to the source at a radial distance of one $L_{e}$. The time origin corresponds to the arrival time $L_{e} / c_{s}$ of the main pulse.

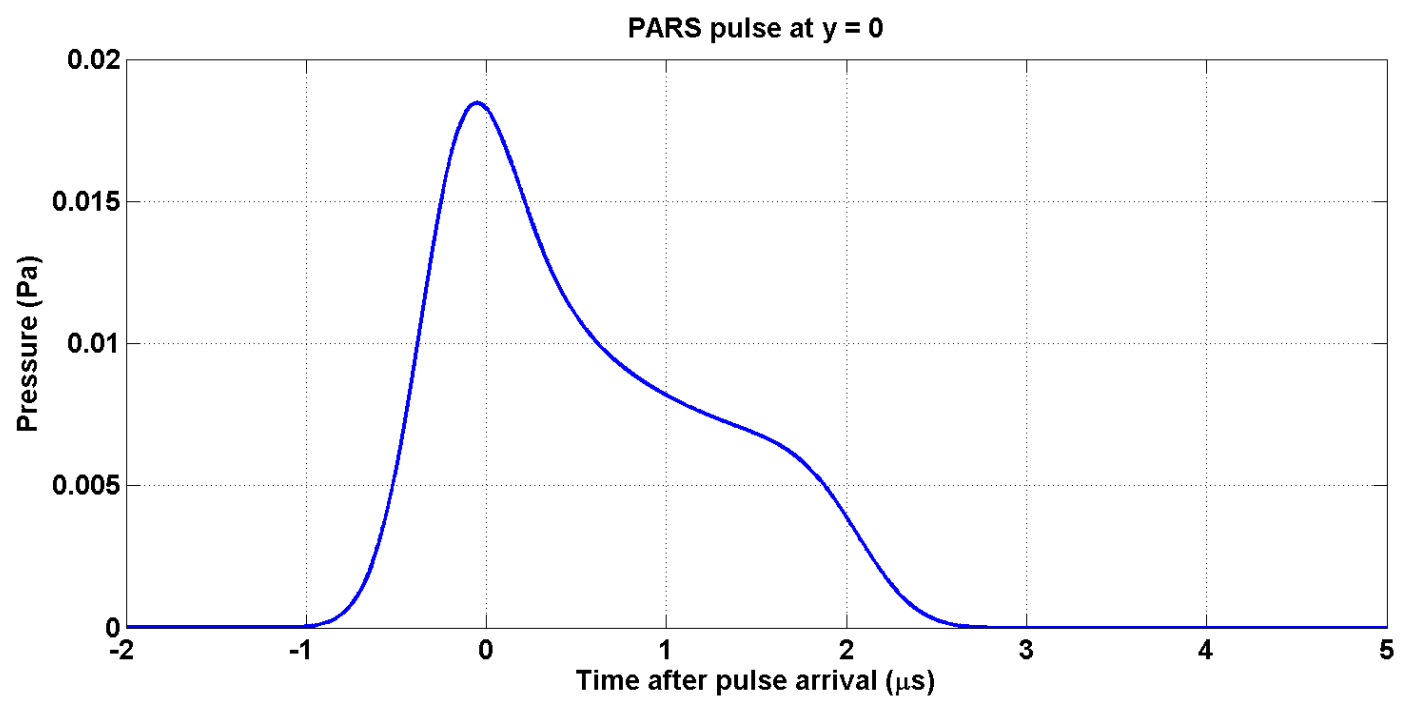

Figure 5: Detail of main pulse in Fig. 4 at $y=0$ showing the significant broadening of the pulse compared to the case without dissipation in Fig. 3. 


\subsection{Instrumentation}

All PARS measurements using either resonant or non-resonant acoustic detection schemes were conducted using one of the experimental setups shown in Figure $6 \mathrm{a}, \mathrm{b}$. Common to both setups is the use of a pulsed Nd:YAG laser operating at $266 \mathrm{~nm}$, a Raman converter (RC) filled with hydrogen $\left(\mathrm{H}_{2}\right)$ gas, and a gas-sample cell equipped with an acoustic detector. The Figure $6 \mathrm{~b}$ experimental setup differs from the other primarily in the optical design, which enables separation and subsequent recombination of the 266 and $299 \mathrm{~nm}$ beams exiting the RC. The Figure $6 \mathrm{~b}$ setup allows one to optimize the temporal and spatial overlap of the beams. Figure $6 a$ and $b$ also utilize different laser triggering schemes; the latter uses an optical chopper and photodiode combination to enable better background subtractions.
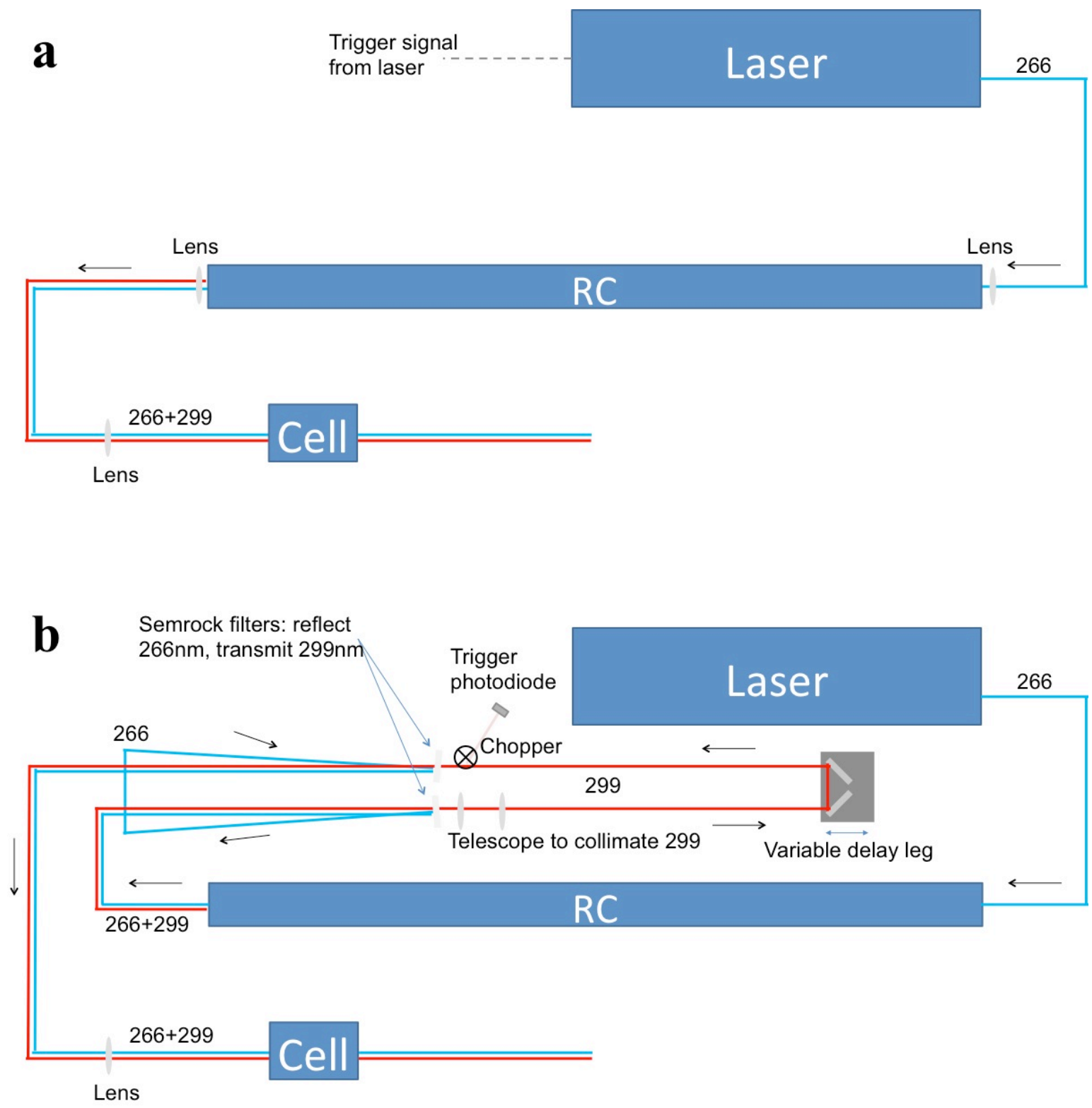

Figure 6a, b: PARS experimental setups. 


\subsection{1 laser}

All measurements were conducted using one of three different lasers with the experimental setups up Figure 6. A Quantronix Eagle Nd:YAG laser (266nm, 50ns pulse duration, $0.5 \mathrm{~mJ} /$ pulse) provided high repetition rate laser pulses (up to $10 \mathrm{~s}$ of $\mathrm{kHz}$ repetition rates) but with low pulse peak power. A Big Sky Ultra $(266 \mathrm{~nm}, 5 \mathrm{~ns}$ pulse duration, $5 \mathrm{~mJ}$ per pulse) provided relatively moderate pulse peak power with up to $20 \mathrm{~Hz}$ repetition rate. A Spectra-Physics (model GCR-130, 266nm, 3-5ns pulse duration, 50mJ per pulse) provided the highest peak power per pulse of all systems but only at $10 \mathrm{~Hz}$ repetition rate. All power values given are the maximum at the laser head.

\subsubsection{Raman converter}

A custom multipass Raman converter for ultraviolet (UV) operation was developed with Light Age. Inc. For all PARS studies, the RC was filled with up to $600 \mathrm{psi}_{2}$ gas. The window and internal RC multipass mirrors were optically coated to pass the generated $\mathrm{H}_{2}$ 1st Stokes $299.05 n m\left[v_{o}-n \times v_{m}=37593.98-(1 \times 4155) \mathrm{cm}^{-1}\right]$ while rejecting the $2 n d$ Stokes $341.48 n m\left[v_{o}-\right.$ $\left.\mathrm{n} \times \mathrm{v}_{\mathrm{m}}=37593.98-(2 \times 4155) \mathrm{cm}^{-1}\right]$ and other higher orders.

\subsection{3 gas-sample cell}

The custom gas-sample cells (two in all) were constructed from vacuum components including a double-ended glass adapter (ID 3-11/16in; cell\#1 length 6.75in, cell\#2 length 10.25in) with two 6in weld-neck flanges, each having through holes for connection to identically sized end flanges. The weld neck and end flanges were sealed using a copper gasket. Both end flanges were designed with a 1.5 in diameter optical window (fused, silica, 0.25 in thick) having anti-reflection (AR) coatings for the UV; each window was sealed against the end flange using indium wire. Each end flange has a brazed (OD 0.25in) stainless steel tube with valve through which gas samples can enter/exit. One of the two end flanges was modified with (i) two vacuum compatible electrical feedthroughs (MS type, 4 pin bulk head) for connecting to the acoustic detectors and (ii) a rail-based support structure (Qioptiq, Nanobench) to support the acoustic detectors and other components (e.g. windowless resonant tube in some experiments). The smaller of the two gas-sample cells is shown in Figure 7. All PARS studies presented herein were performed using neat gases and gas mixtures at a pressure of $25 \mathrm{psi}$.

\subsection{4 acoustic detectors}

Two commercial microphones were used for detecting acoustic signals, a piezo ceramic transducer (Knowles Electronics, BL-21994-000) with a nominal sensitivity of $-49 \mathrm{~dB}$ at $1 \mathrm{KHz}$ (0dB-1V/PA); and a wide band ultrasonic MEMs-based transducer (Knowles Electronics, SPM0404UD5) with a nominal sensitivity of $-42 \mathrm{~dB}$ at $1 \mathrm{KHz}(0 \mathrm{~dB}-1 \mathrm{~V} / \mathrm{PA}) . \quad \mathrm{A} \mathrm{Li}^{+}$ion battery located between the MS bulkhead connector on the gas sample cell and a voltage amplifier supplied power to the acoustic microphone detector.

\subsection{5 gas samples}

Certified gas standards of $\mathrm{H}_{2}$ balanced in $\mathrm{N}_{2}$ or He were purchased from Matheson Tri gas. A custom mass flow-controlled gas mixing system was used for creating calibrated samples of varying dilutions. 


\subsection{6 data acquisition}

The acoustic detector voltage output was amplified using a low noise preamplifier (Stanford Research Systems model SR560) and then fed to a digital oscilloscope (Tektronix model DPO3034GSA) for signal averaging and/or gating depending on the experiment.
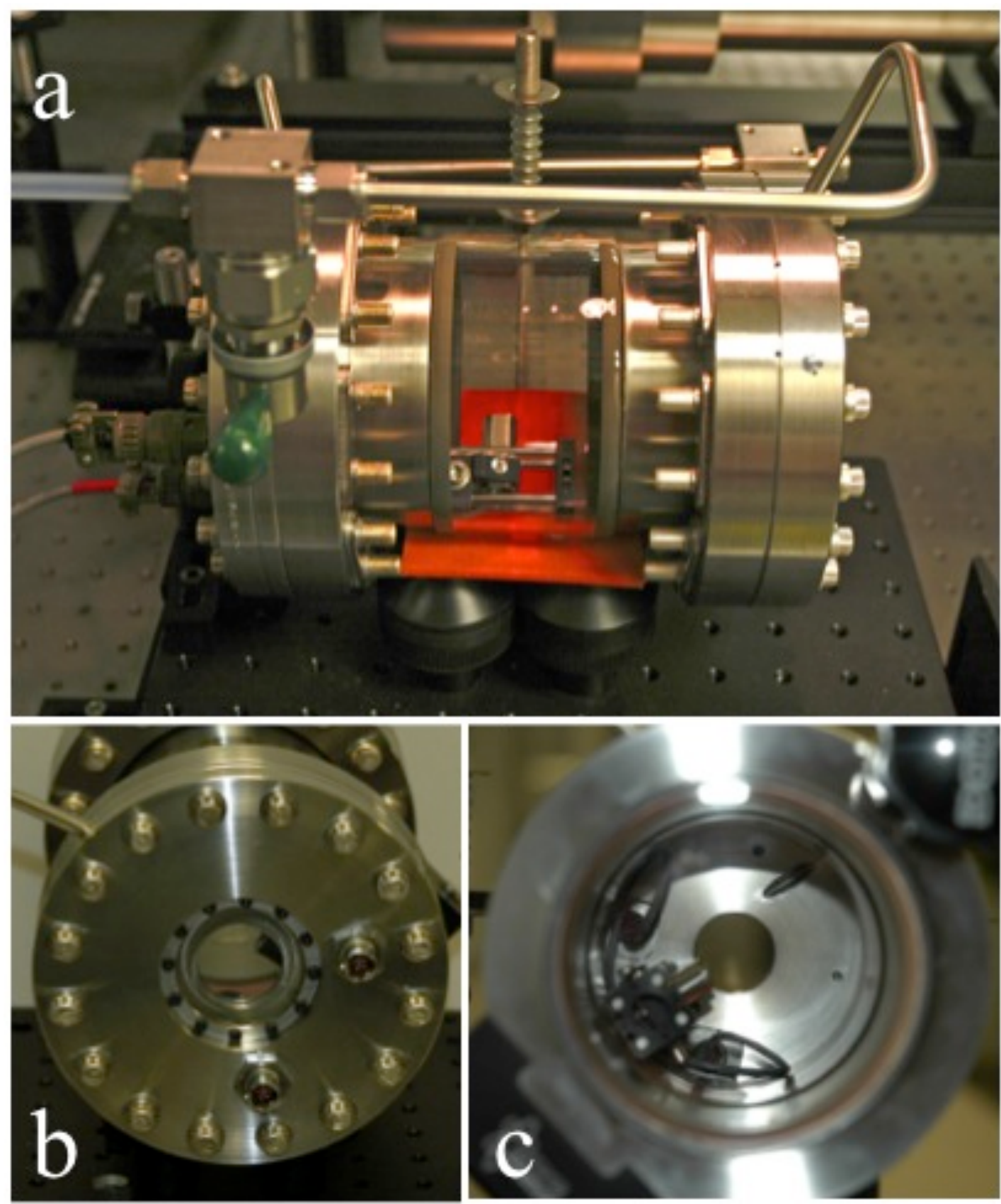

Figure 7: Gas-sample cell showing (a) microphone mounted to internal rail system as viewed through the 2.5in length Pyrex ${ }^{\circledR}$ wall, (b) front view with 1.5in window and two 4 pin electrical connections for the acoustic detector(s), (c) and view from inside the cell. 


\section{Results and Discussion}

The experimental setups illustrated in Figure 6 make use of a Raman converter (RC), which can simply be thought of as another type of source. A Raman converter (also commonly referred to as Raman shifter) is basically a stainless steel tube with optical windows on both ends filled with a high-pressure gaseous medium (e.g. hydrogen, deuterium etc.). The RC is used to extend the tuning range of a laser (e.g. dye, excimer, Nd:YAG, ruby, Ti:Sapphire, and OPO's) via interaction of the pulsed laser beam with the gaseous medium resulting in stimulated Raman emission. Typically, laser pulses are focused into the RC's high-pressure gaseous medium and then recollimated by an integral lens system; single or multipass RC designs are possible. The Raman converter output consists of multiple wavelengths including the incident wavenumber, $v_{o}$ and the Stokes and anti-Stokes laser lines at $v_{0} \pm n \times v_{m}$ where $v_{m}$ corresponds to the frequency of a Raman-active vibration of the scattering molecule (i.e. the gaseous medium) and $n$ takes integral values $1,2,3$,...etc. The Stokes output (i.e., the laser radiation at longer wavelengths than the incident beam) is recollimated and emitted collinearly with the incident beam. The anti-Stokes output lines (i.e. the laser radiation at shorter wavelengths than the incident beam) are also emitted collinearly with the incident beam. When used with a tunable laser pump source, the Raman shifted lines tune in frequency with the pump. Note: the Raman conversion process is not $100 \%$ efficient, thus, unconverted incident laser beam will be transmitted collinear with the Raman converter output line(s). Achieving maximal Raman conversion efficiency requires optimization of the gas, pressure, cell length and focal geometry. For example, a typically energy efficiency for the first Stokes line is $20-40 \%$.

For these studies, a multipass (variable from 1 to 31 passes in increments of 2) capable RC design was developed for the ultraviolet (UV). Unlike most RCs, this design incorporates two internal mirrors to enable internal multipassing; the result is a larger number of passes and fewer losses compared to external multipass RC designs. [Note: the downside of the internal mirror RC design is that each configuration change requires removing and refilling the pressurized gas.] The use of the RC source eliminated the need for a second laser system and enabled the RC to serve as both the pump and probe beams, with an energy difference equivalent to the vibrational energy of the fill gas (i.e. $\mathrm{H}_{2}$ for these studies), required for subsequent PARS experiments. While the use of the RC limited our PARS studies to whatever gas was contained in the RC, it greatly simplified the optical setup, which meant we only had to keep one laser system operational - always a challenge. $\mathrm{H}_{2}$ gas was chosen as the model gas for PARS investigation since $\mathrm{H}_{2}$ has the highest Raman scattering cross section of the homonuclear diatomic gases. The RC was operated with pressurized $\mathrm{H}_{2}$ between 200-600psi; $1^{\text {st }}$ Stokes conversion was maximized over a broad pressure range.

\subsection{Resonant PARS: gas-sample cell}

The scheme for the earliest PARS studies of $\mathrm{H}_{2}$ was based on the Figure 6a setup and the use of a high repetition rate pulsed laser source to drive the acoustic Raman process at a resonance frequency of the custom gas-sample cell. For predicting the cell's cavity resonances, the spatial Helmholtz equation was solved by modeling the smaller of the two gas sample cells as a cylinder with rigid end walls. The predicted resonances (see Table 1) were later validated by a simple experiment in which a function generator was used to drive a speaker placed external to the sample cell while monitoring the acoustic signal amplitude of a microphone 
inside the sealed gas cell. Based on those results, a fixed frequency Nd:YAG laser (Quantronix Eagle) capable of operating in the UV (fourth harmonic, $266 \mathrm{~nm}$ ) at repetition rates covering a broad range of the cavity resonances was chosen. The average and peak powers and spatial profiles of the $266 \mathrm{~nm}$ pulses exiting the laser head were measured at different repetition rates corresponding to the cavity resonances. Results showed the $2.3 \mathrm{kHz}$ repetition rate provided the best beam quality; however, the peak power was low making it necessary to utilize a multipass $\mathrm{RC}$ configuration to achieve higher Raman conversion rates in $\mathrm{H}_{2}$. The process of optimizing the number of passes in the RC was very tedious experimentally since this required removing and refilling the $\mathrm{RC}$ with $\mathrm{H}_{2}$ for each reconfiguration. Figure 8 shows a temporal profile of intensity for the RC input (266nm) and output (266 and 299nm) pulses for a seven pass RC configuration, the most optimal configuration we achieved for producing 266 and $299 \mathrm{~nm}$ pulses with similar power. The time axis origin was set where the oscilloscope triggered on the $266 \mathrm{~nm}$ output signal. The largest conversion occurs at the peak of the $266 \mathrm{~nm}$ input pulse forming a 'valley' in the $266 \mathrm{~nm}$ output (blue trace). As shown in Figure 8 , the wings of the output $266 \mathrm{~nm}$ pump (blue trace) have maximal intensity $\pm^{\sim} 13 \mathrm{~ns}$ from the $299 \mathrm{~nm}$ peak intensity (green trace). Optimizing the number of passes in the RC revealed the importance of having a time delay capability, which resulted in later development of the Figure $6 \mathrm{~b}$ setup.

Table 1. cell cavity resonances

\section{Predicted measured}

1266

1375

1250

1599

1390
1769

1808

1918

2137

2199

2228

2488

2532

2596

2828

2831

2972

3016

1750

1850

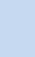

\begin{tabular}{|ll|}
2199 & \\
2228 & 2400 \\
\hline 2488 & 2560 \\
\hline 2532 & \\
\hline 2596 & \\
2828 & \\
\hline 2831 & \\
\hline 2972 & 3270 \\
\hline 3016 & \\
\hline
\end{tabular}

(n)

we had difficulty stabilizing the pump and probe beams exiting the RC were much lower in power than predicted, possibly due to scattering or thermal effects. Even so, we were eventually able to perform a series of 
controlled studies using neat $\mathrm{N}_{2}$ and different concentrations of $\mathrm{H}_{2}$ in $\mathrm{N}_{2}$. For all measurements, the acoustic detector signal output was fed to a preamplifier with some frequency filtering and then the oscilloscope for signal averaging (typically 128 or more) to attenuate both the random noise and periodic but asynchronous noise leaving only the laser driven noise (thus improving the signal-to-noise $(\mathrm{S} / \mathrm{N})$ ratio. The frequency content of our signal was analyzed by taking a fast-Fourier transform (FFT), which was performed in post-processing using a Matlab routine. Figures 9 and 10 show representative examples of the time and frequency domain data for $\mathrm{N}_{2}$ neat $\left(0_{p p m} \mathrm{H}_{2}\right)$ and $1000 \mathrm{pm}_{v} \mathrm{H}_{2}$ in $\mathrm{N}_{2}$, respectively. The top spectrum in each figure is the amplified averaged signal followed by filtering (mid) and then power spectrum (bottom) [Note: laser driven signals must consist purely of frequencies matching the laser rep rate and higher harmonics. The filter we implemented blocked everything but the laser harmonics. It was similar to a bandpass filter with many discreet pass bands]. Our detection methodology was consistent with what had been discussed in the literature and was superior to lock-in detection in several regards. As shown in the Figure 11 power spectrum for the $1^{\text {st }}$ and $2^{\text {nd }}$ laser/gas cell harmonics at 3 different $\mathrm{H}_{2}$ concentrations balanced in $\mathrm{N}_{2}$, we observed some features in our power spectra that scaled with concentration as expected (similar to data reported in the literature[8]) and some that did not scale. We were unable to conclusively attribute any of the features to PARS and believe the power spectrum results to be random fluctuations. Another chamber resonance $(1.25 \mathrm{kHz})$ was investigated and produced similar results as described above. Ultimately, we abandoned the high repetition rate laser having concluded that the pump and probe beams exiting the RC were insufficient to drive the PARS process to detectable levels in the gas cell.

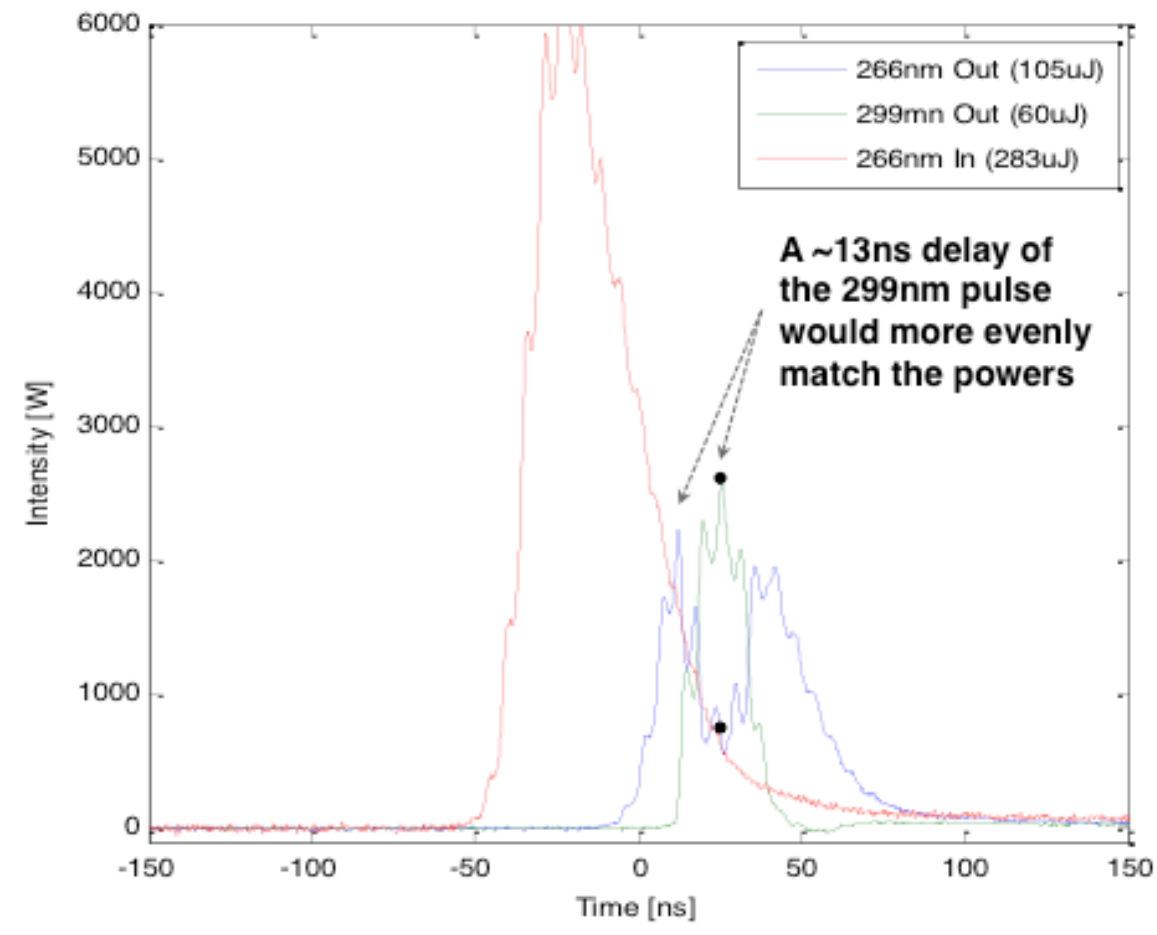

Figure 8: Temporal intensity profile for the RC input $(266 \mathrm{~nm}$, red trace) and output pulses (266nm, blue trace; and 299nm; green trace) for a seven pass RC configuration. 

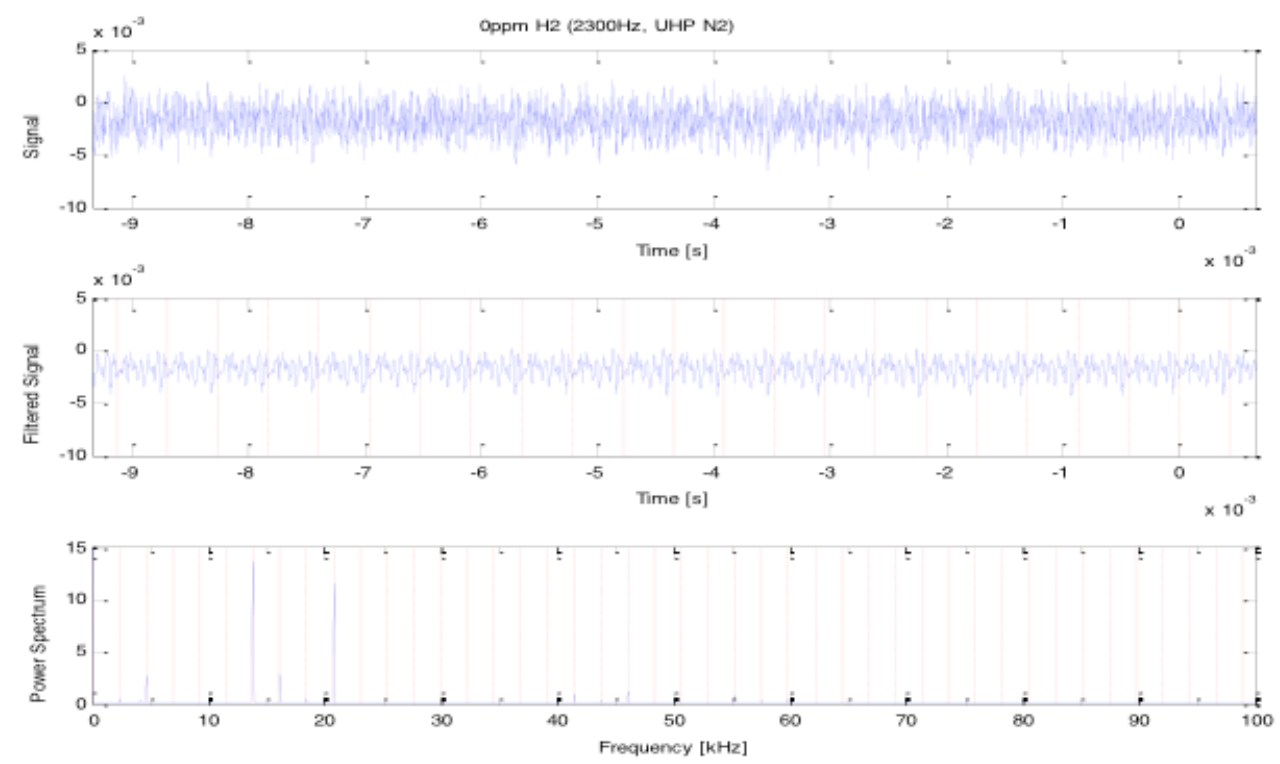

Figure 9: Time (top and mid) and frequency domain (bottom) data for $\mathrm{N}_{2}$ neat $\left(0 p \mathrm{pm}_{\mathrm{v}} \mathrm{H}_{2}\right.$ in $\mathrm{N}_{2}$ ). The data was acquired using a laser repetition rate of $2.3 \mathrm{kHz}$, which matched a cavity resonance (see Table 1) of the gas-sample cell. For the filtered signal (mid), the red dashed lines show each laser pulse and in the power spectrum (bottom) each harmonic of the laser.
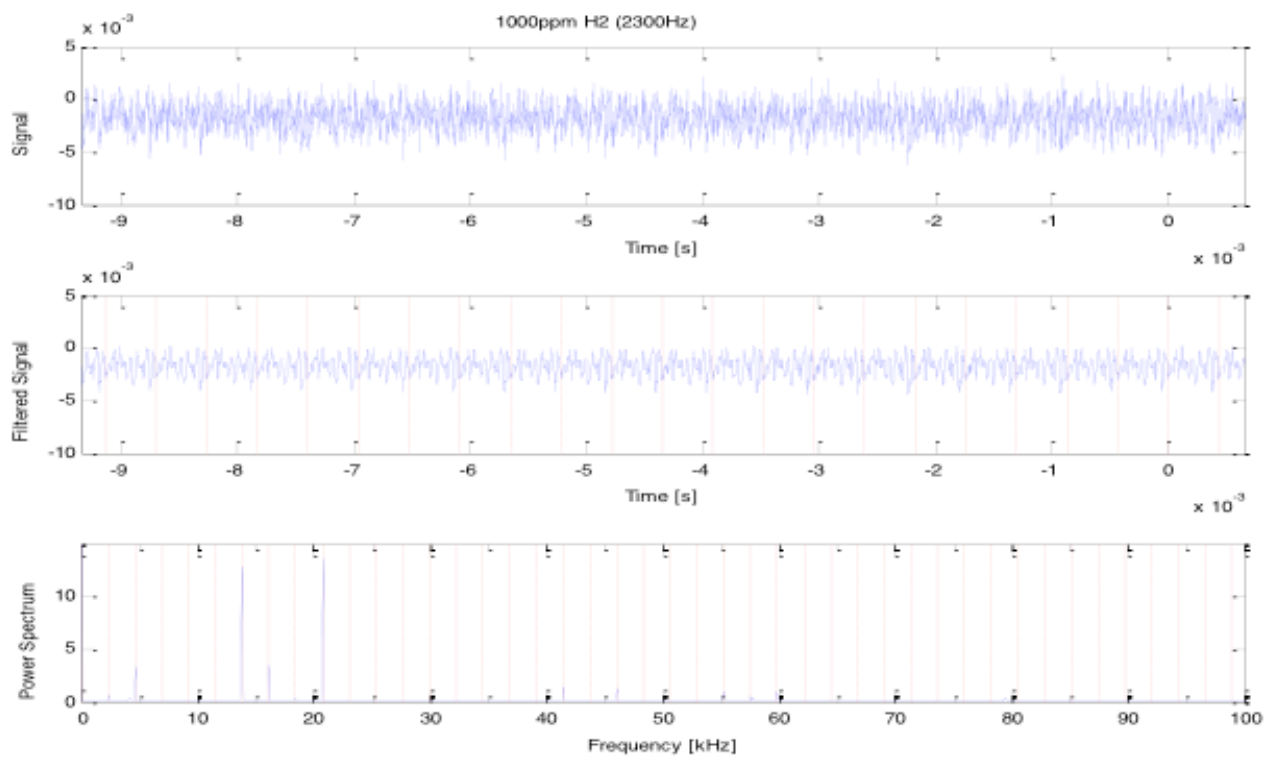

Figure 10: Time (top and mid) and frequency domain (bottom) data for $\mathrm{N}_{2}$ neat (1000ppm $\mathrm{H}_{2}$ in

$\mathrm{N}_{2}$ ). The data was acquired using a laser repetition rate of $2.3 \mathrm{kHz}$, which matched a cavity resonance (see Table 1) of the gas-sample cell. The red dashed lines are analogous to those in

Figure 9. 


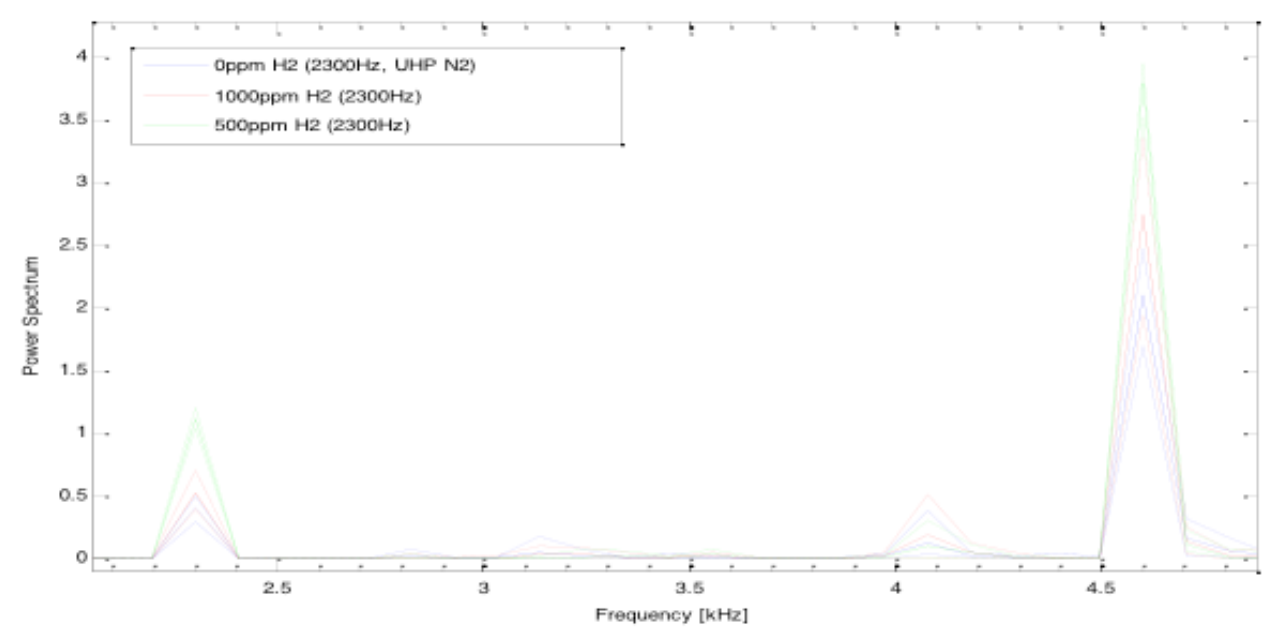

Figure 11: Power spectrum results (from Figure 9 and 10 and including additional concentration data at 500ppm $\mathrm{H}_{\mathrm{V}}$ ) for the $1^{\text {st }}$ and $2^{\text {nd }}$ laser/gas cell harmonics at 3 different $\mathrm{H}_{2}$ concentrations: Oppm $\mathrm{H}_{2}$ (i.e. $\mathrm{N}_{2}$ neat), $500 p p m_{v} \mathrm{H}_{2}$ in $\mathrm{N}_{2}, 1000 \mathrm{pm}_{\mathrm{v}} \mathrm{H}_{2}$ in $\mathrm{N}_{2}$. The data was acquired using a laser repetition rate of $2.3 \mathrm{kHz}$, which matched a cavity resonance (see Table 1 ) of the gas-sample cell.

\subsection{Resonant PARS: windowless resonant tube within gas-sample cell}

Oki and coworkers reported[8] PARS detection of $\mathrm{H}_{2}(3.4 \mathrm{ppm})$ utilizing the second harmonic of an $\mathrm{Nd}$ :YAG laser pulsing at a resonant frequency of a small windowless acoustic resonator and microphone assembly placed inside their gas-sampling cell. They reported an increase in the PARS S/N when the acoustic energy was concentrated in a few dominant modes of the windowless acoustic resonator tube, which corresponded to $1-2 \mathrm{kHz}$ in their setup. Though many resonances appeared in the power spectrum, Oki reported that most of them contained no PARS signal component.

Similarly, we attempted PARS measurements using a windowless acoustic resonator tube (a fused silica tube, $120 \mathrm{~mm}$ length, $4.75 \mathrm{~mm}$ ID, $9.0 \mathrm{~mm}$ OD) and higher sensitivity acoustic detector (Knowles Electronics, SPM0404UD5) assembly placed within our custom gas-sampling cell as shown in Figure 12. Rather than located at the center, the acoustic detector was placed at a $45^{\circ}$ angle just below one end of the tube. We evaluated other laser systems and eventually replaced the high repetition rate laser with one (Spectra Physics GLD-130, 266nm, 10Hz, 3-5ns pulse duration, $50 \mathrm{~mJ}$ per pulse) operating at a significantly higher peak power. The RC was also modified for a single pass only. The laser beam was collimated rather than focused into the RC, since focusing resulted in an additional concentric ring of light at the RC output indicating undesirable 4-wave mixing effects. The Figure $6 \mathrm{~b}$ experimental setup was also adopted to allow separation and subsequent recombination of the RC $266 \mathrm{~nm} 299 \mathrm{~nm}$ output. This enabled beam quality improvement as well as the ability to attenuate either the 266 and/or $299 \mathrm{~nm}$ beams. The addition of an optical chopper and photodiode combination also enabled better background subtraction. 


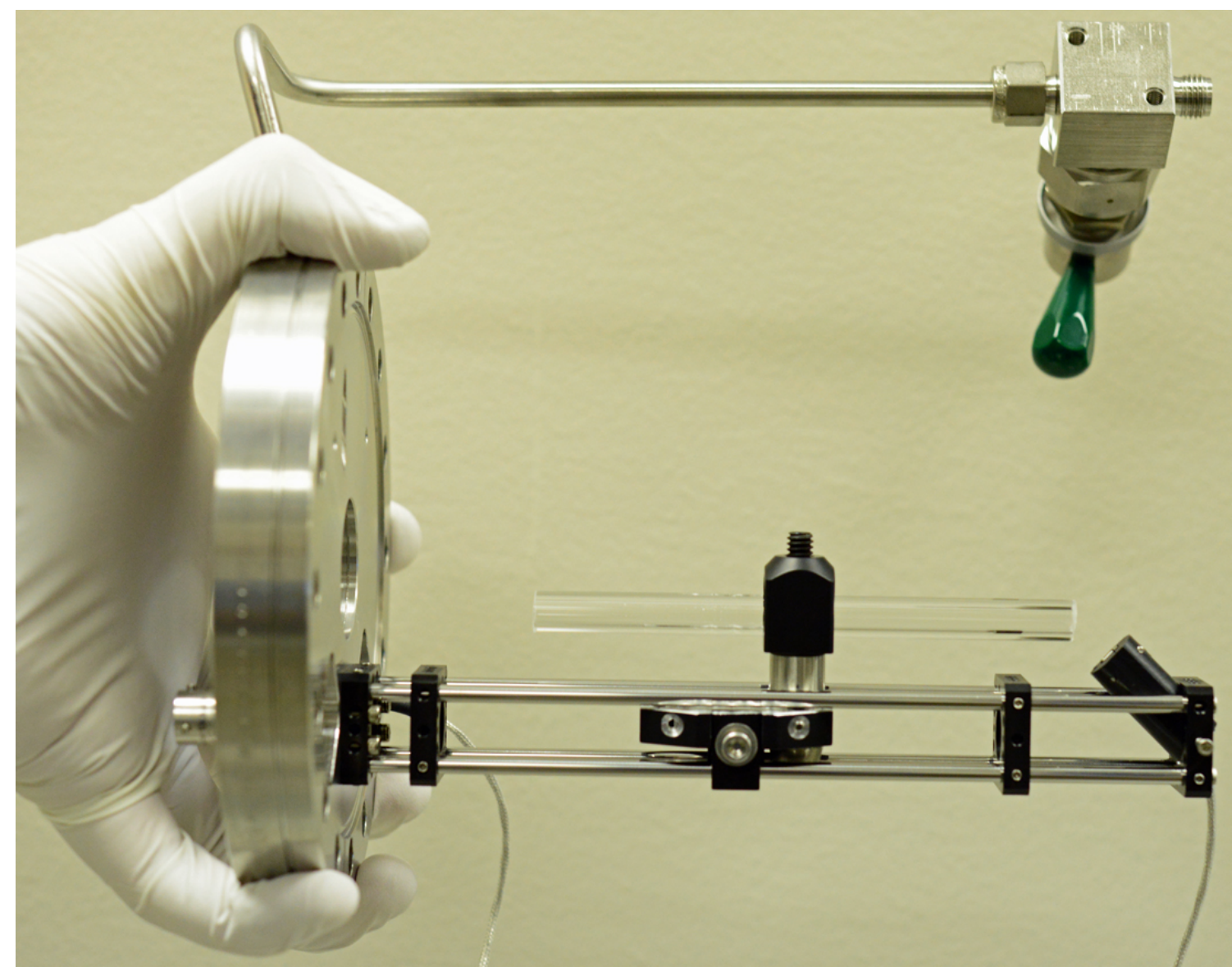

Figure 12: Gas-sample cell end flange showing the windowless resonant (glass) tube mounted on the rail system along with the acoustic detector (far right) angled toward one end of the tube.

Figure 13 (top) shows the average acoustic waveform generated by a single $266 \mathrm{~nm}$ pulse in $\mathrm{N}_{2}$ neat and the corresponding power spectrum (bottom). Peaks in the power spectrum are attributed to the first-order resonant tube frequency $(1375 \mathrm{~Hz})$, harmonics of the resonant tube $(2750 \mathrm{~Hz}, 4125 \mathrm{~Hz})$ and potentially a resonance of the gas-sample cell $(4750 \mathrm{~Hz})$. The acoustic waveform in Figure 13 does not contain a PARS signal since the sample contained no $\mathrm{H}_{2}$ gas; however, there is significant acoustic signal solely attributed to noise and unwanted background phenomena. A similar experiment performed using $1000 \mathrm{ppm}_{v} \mathrm{H}_{2}$ in $\mathrm{N}_{2}$ shows a similar acoustic waveform although somewhat attenuated (see figure 14 top). We were unable to ascertain the exact reason for the acoustic intensity difference but believe fluctuations in the laser power or beam alignment are candidate reasons. Even so the difference is only a factor of $2-3$. We still remained puzzled as to why the intensity of the $4750 \mathrm{~Hz}$ peak in the power spectra of Figure 13 and 14 (bottom) differ significantly. We did observe that the peak intensities in the power spectrum were very sensitive to laser alignment. It is possible that the laser was focusing slightly differently within the resonant tube due to laser drift. Even so, we were not able to confirm that this frequency contained any PARS signal.

Although slight variations of resonant PARS experiments were also investigated, we were unable to conclusively measure PARS of $\mathrm{H}_{2}$ using resonant approaches. The high acoustic 
background signals (i.e. noise) masked any PARS signal present. A more fundamental understanding of our noise source(s) was needed.
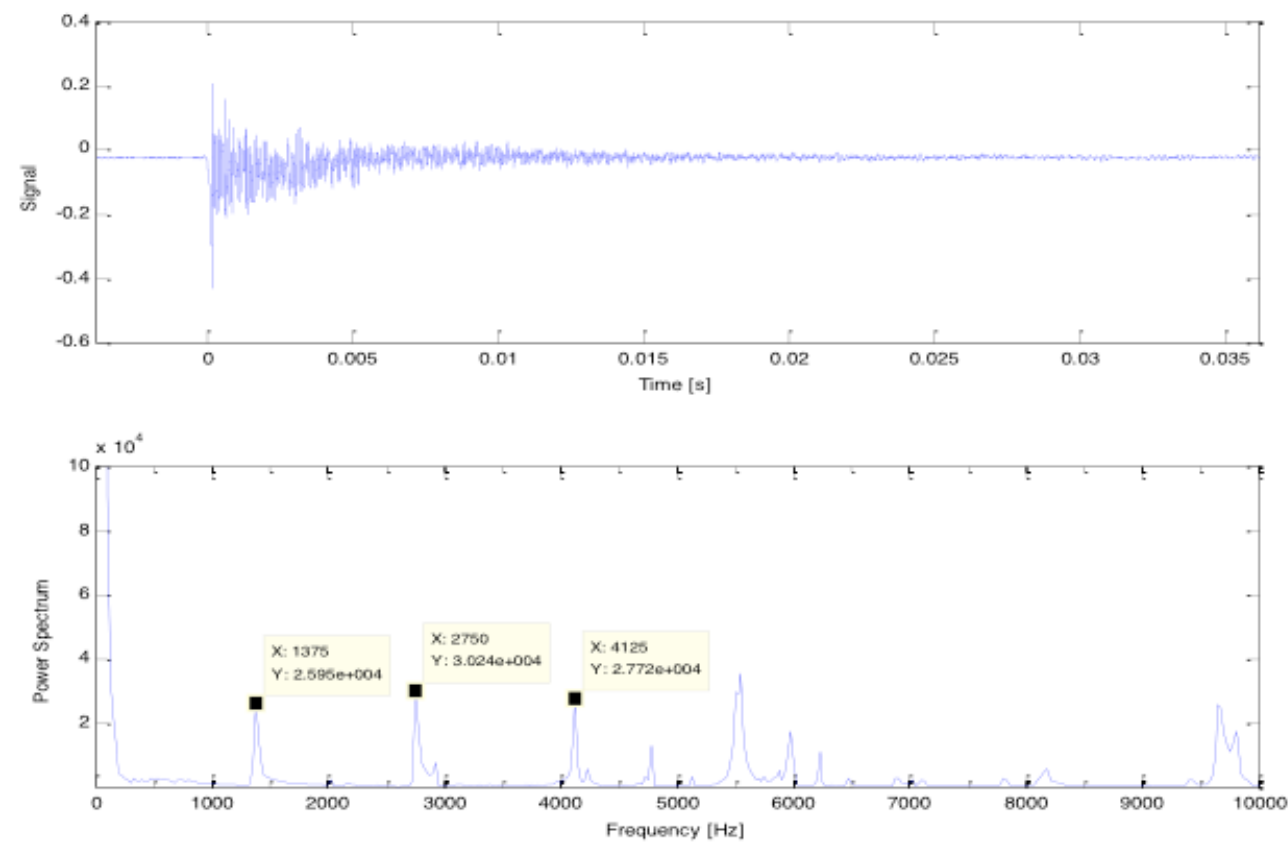

Figure 13: Time (top) and frequency domain (bottom) data for $\mathrm{N}_{2}$ neat. The data was acquired using a windowless acoustic resonator tube and acoustic detector assembly within a custom gas-sampling cell.
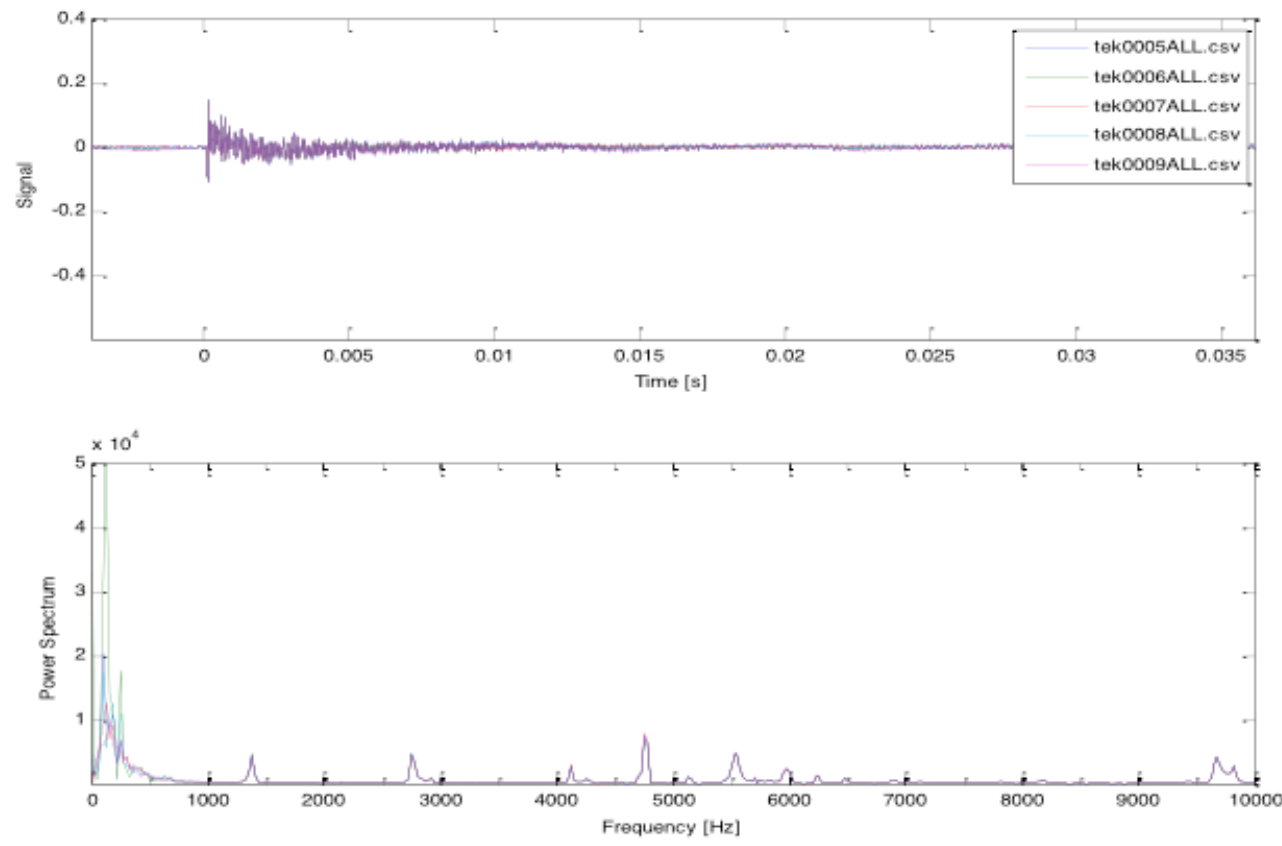

Figure 14: Time (top) and frequency domain (bottom) data for $1000 \mathrm{ppm}_{v}$ in $\mathrm{N}_{2}$. The data was acquired using a windowless acoustic resonator tube in a gas-sample cell. 


\subsection{Non-resonant PARS}

Although resonant detection approaches are likely more sensitive methods for PARS, these tend to blur all noise. The non-resonant approach is better for studying the noise, provided the acoustic detector has sufficient temporal resolution. The Knowles acoustic detector (model number SPM0404UD5) discussed in the previous section was not only more sensitive for low frequencies but also had the added benefit of greater bandwidth with a peak sensitivity in the $40-50 \mathrm{kHz}$ region. As simple time-bandwidth considerations would suggest, acquisition of higher acoustic frequencies produces better temporal resolution. As shown in the representative acoustic waveform of Figure 15 acquired with the setup described in the previous section but without the windowless resonant tube present, it became possible to clearly identify RF noise occurring before any possible acoustic signal could reach the acoustic detector. It also became possible to identify acoustic noise generated from gas cell walls and windows, which arrived at the acoustic detector well after the onset of signal originating from the laser focus. (The acoustic detector was intentionally positioned as close as possible to the laser focus and far from the gas-sample cell windows and walls). Most importantly, the non-resonant approach with higher bandwidth acoustic detector revealed the presence of an acoustic signal generated at the laser focus that was not a PARS signal. This was largely unexpected and highly undesirable.

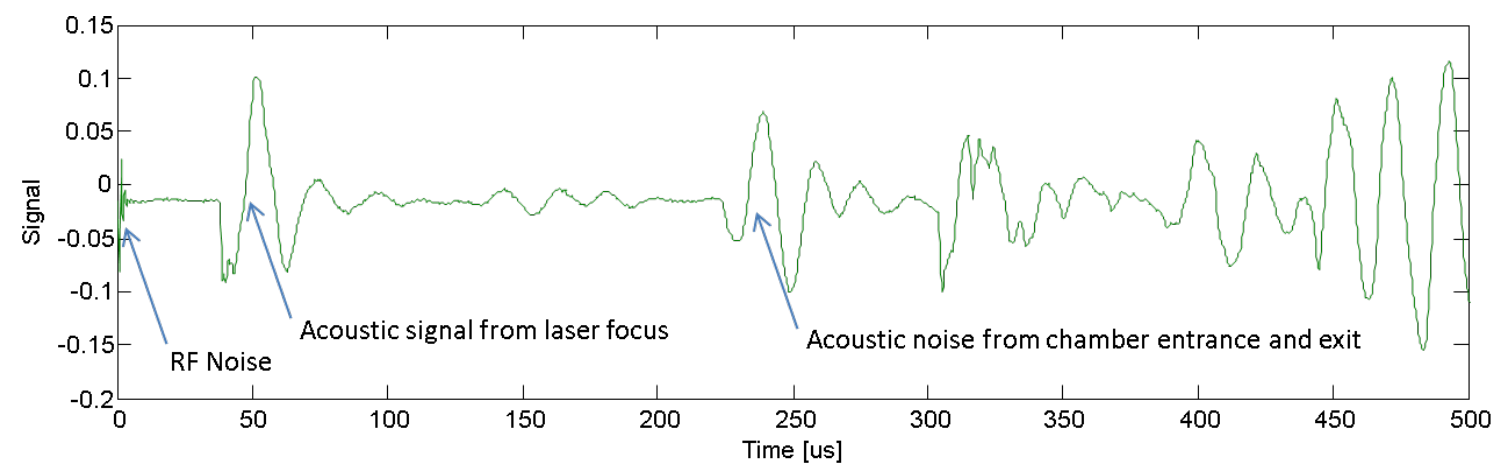

Figure 15: Time domain data for a non-resonant PARS experiment utilizing a high bandwidth microphone. With the improved temporal resolution, several distinct noise sources are apparent.

The unknown acoustic signal generated at laser focus (i.e. noise) was detected whether using the combined $266 \mathrm{~nm}$ and $299 \mathrm{~nm}$ laser beams or only the $266 \mathrm{~nm}$ focused into the gas-sample cell. This unwanted background could be produced in ambient air, $\mathrm{N}_{2}$ neat, $\mathrm{Ar}$ neat, $\mathrm{He}$ neat and different concentrations of $\mathrm{H}_{2}$ in $\mathrm{N}_{2}$ or $\mathrm{He}$ (although we primarily worked with $\mathrm{H}_{2}$ balanced in $\mathrm{N}_{2}$ ). The arrival time and frequency content of the signal varied slightly with gas, but this was attributed primarily to differences in sound speed. In real time, we could vary the separation between the acoustic detector and laser focus and watch the arrival time of this signal change accordingly (due to finite sound speed). The fact that the signal originated from the laser focus alone and not uniformly along the length of the laser beam suggested that a nonlinear process was involved. This was confirmed with laser power studies, as shown in Figure 16, where the detected acoustic signal (a voltage proportional to sound pressure) is plotted versus time (note log scales). The data is well fit by a curve proportional to laser pulse 
energy raised to the 2.4 power; however, our confidence in that value is not high. Given uncertainties in the laser power and acoustic measurements, we merely claim an exponent $\geq 2$. The acoustic power is proportional to the pressure squared, thus the acoustic power varies with the laser energy (and laser intensity) to the $\geq 4$ th power. This suggests a $\geq 4$-photon process. Four $266 \mathrm{~nm}$ photons have a combined energy of $18.6 \mathrm{eV}$. The ionization potential of $\mathrm{N}_{2}$ is 14.5eV. Multiphoton ionization (MPI) of $\mathrm{N}_{2}$ is consequently a possibility. Any laser energy absorbed by the gas (whether due to MPI or other processes) will eventually thermalize, generate heat and produce a change in pressure and thus an acoustic signal. In fact, we know from literature that photoacoustic detection is one method of measuring resonance enhanced multiphoton ionization (REMPI). MPI could be the source of the acoustic signals generated at laser focus, but this remains unconfirmed. If the noise is indeed MPI of $\mathrm{N}_{2}$ then working with UV excitation, compared to visible excitation, is detrimental since fewer photons are required (e.g. 4 versus 7 in the case of $266 \mathrm{~nm}$ versus $532 \mathrm{~nm}$ light). It is worth noting that PARS is a two-photon process regardless of wavelength.

This background signal from the laser focus is probably the biggest single obstacle to successful UV PARS detection. It is sufficiently large and variable that any underlying PARS signal is obscured. Due to the fact that this background originates from the same point in time and space as our desired PARS signal and is expected to have overlapping frequency content, we have been unable to filter or suppress it effectively. We have implemented schemes that allow us to near simultaneously acquire two signals: one where a PARS signal should be present and one without (with the unwanted background present in both cases). Unfortunately, the difference (background subtraction) in these signals has never appeared statistically significantly; furthermore the subtraction only adds more noise to the resulting spectrum. We attempted to optically detect photons generated by the PARS process but laser-induced fluorescence from cell windows was an overwhelming interferent.

Acoustic Signal vs Laser Power Density

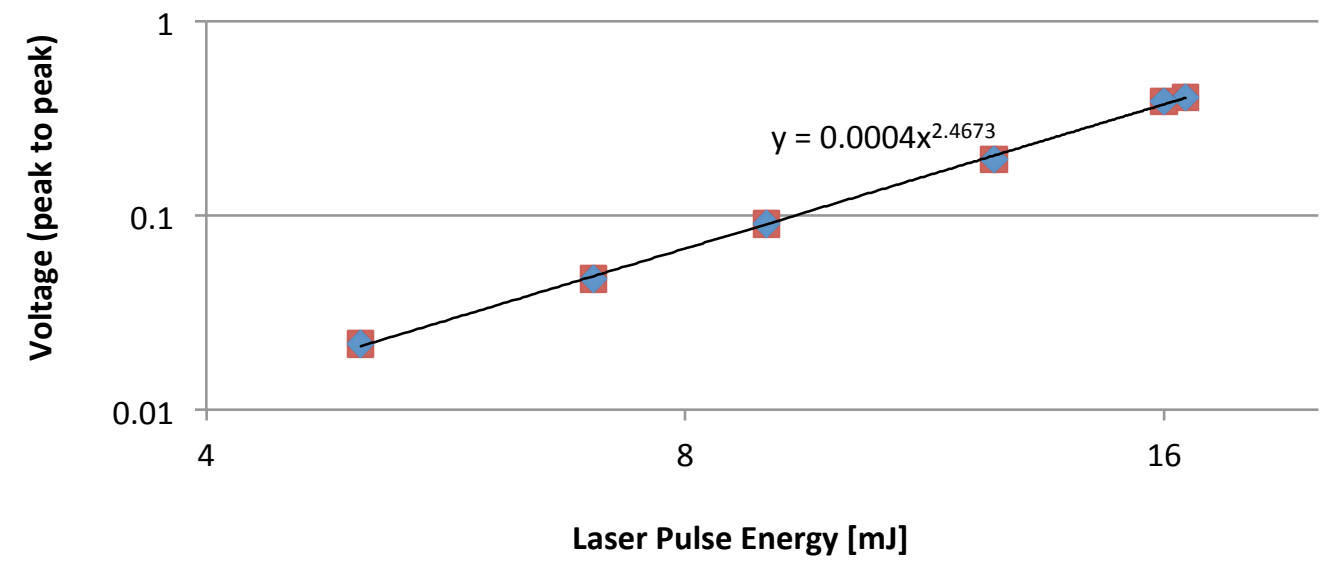

Figure 16: The detected acoustic signal versus laser pulse energy (which is proportional to intensity) for $\mathrm{N}_{2}$ neat. 


\section{Conclusions}

To summarize, our efforts and findings are as follows: we analyzed the theoretical system performance using known PARS theory coupled with an acoustic detector model to estimate the expected signal-to-noise ratio (SNR). The system model comprised a mathematical model of the Raman process leading to a prediction of the temperature change in the active region; a thermoacoustic gas prediction of the radiated pressure field (amplitude and pulse shape); and the receiver response for an acoustic microphone, including a simple model of the receiver circuitry (filters, integrators, etc.). Based on the PARS experimental parameters in Appendix $\mathrm{B}$, the model predicted a PARS signal with pressure peak of $7 \mathrm{~Pa}$ and duration slightly longer than $2 \mathrm{~ms}$ at a distance of $7 \mathrm{~mm}$ from the focal spot when acoustic dissipation is not included. An analytical model of a PARS signal with acoustic dissipation was constructed but the numerical calculation is limited to gains of $<1 \%$ of the experimental value. For these lower gains, the model predicts spreading of the signal.

For laboratory PARS studies, a multipass (variable from 1 to 31 passes in increments of 2) Raman converter was developed with an outside vendor for UV pulsed excitation. Unlike most Raman converters, this design incorporated two internal mirrors enabling internal multipassing; the result was a larger number of passes and fewer losses compared to external multipass RC designs. The use of the Raman converter source eliminated the need for a second laser system and greatly simplified the experimental setups. $\mathrm{H}_{2}$ gas was chosen as the model gas for PARS investigation since $\mathrm{H}_{2}$ has the highest Raman scattering cross section of the homonuclear diatomic gases. The Raman converter was successfully operated with pressurized $\mathrm{H}_{2}$ between 200-600psi, producing efficient $1^{\text {st }}$ Stoke Raman conversion. To our knowledge, we were the first to demonstrate high repetition rate UV Raman conversion of $\mathrm{H}_{2}$ using a multipass Raman converter.

We constructed the first experimental setups based on UV excitation of $\mathrm{H}_{2}$ in $\mathrm{N}_{2}$ for resonant and non-resonant PARS to validate the system model but were unable to confirm a detectable PARS signal due in part to a non-PARS acoustic signal generated simultaneously at the laser focus. This unwanted background could be produced in ambient air, $\mathrm{N}_{2}$ neat, $\mathrm{Ar}$ neat, $\mathrm{He}$ neat and different concentrations of $\mathrm{H}_{2}$ in $\mathrm{N}_{2}$ or $\mathrm{He}$. The fact that the signal originated from the laser focus alone and not uniformly along the length of the laser beam suggested that a nonlinear process was involved, which was confirmed with laser power studies using higher temporal resolution acoustic measurements of $\mathrm{N}_{2}$ neat and $\mathrm{H}_{2}$ in $\mathrm{N}_{2}$. The acoustic power was found to vary with the laser energy (and laser intensity) to the $\geq 4$ th power, suggesting a $\geq 4$-photon process. Given the ionization potential of $\mathrm{N}_{2}(14.5 \mathrm{eV})$, we have tentatively concluded that multiphoton ionization (MPI) of $\mathrm{N}_{2}$ is a major source of noise that prevented a detectable PARS signal of $\mathrm{H}_{2}$ at the UV excitation wavelengths and laser powers used in our studies. 


\section{References}

[1] J. J. Barrett. Photoacoustic Raman Spectroscopy of Gases. Appl. Spect. Rev., 21(4):419-464, 1985.

[2] Philip M. Morse and K. Uno Ingard. Theoretical Acoustics. Princeton University Press, Princeton, NJ, 1968.

[3] Leonid M. Lyamshev. Radiation Acoustics. CRC Press, Boca Raton, FA, 2004.

[4] S.H. Yonak and D.R. Dowling. Gas-phase generation of photoacoustic sound in an open environment. J. Acoust. Soc. Am., 114(6):3167-3178, 2003.

[5] K.K. Das and Y.V. Rostovtsev and K. Lehmann and M.O. Scully. Thermodynamic and noise considerations for the detection of microscopic particles in a gas by photoacoustic Raman spectroscopy. Opt. Comm., 246:551-559, 2005.

[6] A. B. Bhatia. Ultrasonic Absorption. Dover Publications, New York, NY, 1985.

[7] I. S. Gradshteyn and I. M. Ryzhik. Table of Integrals, Series, and Products. Academic Press, New York, NY, 1980.

[8] Y. Oki, N. Kawada, Y. Abe, M. Maeda,. Non-linear Raman Spectroscopy without tunable laser. Opt. Comm., 161, 57-62, 1999. 


\section{Appendix A}

In the text the pressure field is determined by combinations of the function $f(x, y)$ whose definition is given by equation (25) and repeated below:

$$
f(x, y)=\int_{1}^{y} \frac{d t}{\sqrt{t^{2}-1}} e^{x t}
$$

By a change of variables we can put this in the form

$$
f(x, y)=e^{x y} \sqrt{\frac{y-1}{y+1}} \int_{0}^{1} \frac{d u}{\sqrt{(1-u)\left(1-\frac{y-1}{y+1} u\right)}} e^{-x(y-1) u} .
$$

The integral can be found in the reference Gradshteyn \& Ryzhik[7], equation 3.385:

$$
f(x, y)=e^{x y} \sqrt{\frac{y-1}{y+1}} B\left(1, \frac{1}{2}\right) \Phi_{1}\left(1, \frac{1}{2}, \frac{3}{2}, \frac{y-1}{y+1},-x(y-1)\right),
$$

where $B$ is the Euler integral of the first kind and $\Phi_{1}$ is the degenerate hypergeometric series in two variables. Though the expression is exact it gives no information on the expected shape of the waveform.

As noted in the text, if we are interested in calculating the pulse at distances where the acoustic travel time is much greater than the time scale of the Raman scattering, we only need the asymptotic form of $f(x, y)$ for large $x$. In this limit the exponential factor in the integrand dominates the algebraic factor except near the endpoint $t=1$ where $1 / \sqrt{t^{2}-1}$ is singular. However, this singularity is integrable and can be eliminated by first performing integration by parts using

$$
\int \frac{d t}{\sqrt{t^{2}-1}}=\ln \left(1+\sqrt{t^{2}-1}\right)
$$

The new expression for $f$ is

$$
f(x, y)=e^{x y}\left[\ln \left(y+\sqrt{y^{2}-1}\right)-x \int_{0}^{y-1} \ln \left(y-s+\sqrt{(y-s)^{2}-1}\right) e^{-x s} d s\right]
$$

where we also applied a change of integration variable, $t=y-s$. The logarithm term in the integrand is positive, bounded, and monotonically decreasing from $\ln \left(y+\sqrt{y^{2}-1}\right)$ at $s=0$ to zero at $s=y-1$. For large $x$ the exponential factor in the integrand decreases rapidly (assuming $x(y-1)>>1$ ) so that the primary contribution to the integral occurs near the lower endpoint $s=0$. Thus we can expand the logarithmic term in a power series near $s=0$, then perform the integral term-by-term to obtain a power series in $1 / x$ for the asymptotic behavior of $f$ in the limit $x(y-1) \rightarrow \infty$. The power series for the logarithmic term is

$$
\ln \left(y-s+\sqrt{(y-s)^{2}-1}\right)=\ln \left(y+\sqrt{y^{2}-1}\right)-\frac{s}{\sqrt{y^{2}-1}}-\frac{y s^{2}}{2\left(y^{2}-1\right)^{3 / 2}}+0\left(s^{3}\right) .
$$

Substituting this expansion into the integrand and setting the upper limit to $\infty$ (with exponentially small error) we obtain the asymptotic expression of $f(x, y)$ for $x(y-1) \rightarrow \infty$ : 


$$
f(x, y) ; \frac{e^{x y}}{x \sqrt{y^{2}-1}}\left[1+\frac{y}{x\left(y^{2}-1\right)}+0\left(1 / x^{2}\right)\right]
$$

The previous expression is valid for $x(y-1)$ ? 1 , which is the typical case for the pressure field far from the source region. However, for the pressure field in a plane perpendicular to the source region $(z=0)$ we must evaluate $f(x, y)$ for $y=R / r=1$. It is obvious from the definition that $f(x, y) \rightarrow 0$ as $y \rightarrow 0$. However, the asymptotic expression derived previously (equation (41) is singular as $y \rightarrow 0$. We need another large $x$ expression for $f$ that is valid for $x$ ? 0 but with $x(y-1)=1$. This is easily obtained after changing the integration variable in the definition to obtain

$$
f(x, y)=e^{x} \int_{0}^{y-1} \frac{e^{x s}}{\sqrt{2 s(1+s / 2)}} d s .
$$

Since $x(y-1)=1$, we can replace the exponential factor and $(1+s / 2)$ term with unity, perform the resulting integral, and obtain the approximation

$$
f(x, y) ; \sqrt{2(y-1)} e^{x}, \quad x \rightarrow \infty, x(y-1) \rightarrow 0
$$

We now have two asymptotic expressions valid for large $x$, one for $x(y-1)$ ? 1 and the other for $x(y-1)=1$. There is no simple expression for the intermediate limit with $x(y-1)=0(1)$. However, if we are interested mostly in the qualitative behavior of $f(x, y)$ we can combine the two asymptotic limits into a single expression that incorporates both behaviors:

$$
f(x, y) ; \frac{e^{x y}}{\sqrt{x}} \sqrt{\frac{x\left(y^{2}-1\right)}{1+x^{2}(y-1)^{2}}} .
$$

This expression smoothly transitions between the two asymptotic limits and is used to generate the plots in the paper. 
Appendix B

PARS model parameters

\begin{tabular}{l|l}
\hline Pump wavelength $\lambda_{p}$ & $266 \mathrm{~nm}$ \\
\hline Stokes wavelength $\lambda_{p}$ & $299 \mathrm{~nm}$ \\
\hline Refractive indices $n_{p}, n_{s}$ & 1 \\
\hline Linewidth $\Gamma$ & $189 \mathrm{GHZ}$ \\
\hline Pump intensity $I_{p \theta}$ & $1.1 \times 10^{15} \mathrm{~W} / \mathrm{m}^{2}$ \\
\hline Stokes intensity $I_{s \theta}=\mathbf{2 0} \% I_{p \theta}$ & $8.8 \times 10^{14} \mathrm{~W} / \mathrm{m}^{2}$ \\
\hline Collisional rate $k_{c}^{1}$ & $10^{3} \mathrm{~s}^{-1}$ \\
\hline Length of focal region $L_{e}$ & $7 \mathrm{~mm}$ \\
\hline Diameter of focal region $D_{m}$ & $4 \times 10^{-5} \mathrm{~m}$ \\
\hline Population density $N_{T}\left(\mathrm{H}_{2}\right.$ in $\left.\mathrm{N}_{2}\right)$ & $2.5 \times 10^{26} \mathrm{~m}^{-3}$ \\
\hline Pulse duration $t_{l}$ & $5 \mathrm{~ns}$ \\
\hline Sound speed $c_{s}$ & $330 \mathrm{~m} / \mathrm{s}$ \\
\hline Cross-section $(d o f d \Omega)$ & $1 \times 10^{-34} \mathrm{~m}^{2}$ \\
\hline
\end{tabular}

